\title{
WARM WATER VAPOR AROUND SAGITTARIUS B2 ${ }^{1}$
}

\author{
José Cernicharo, Javier R. Goicoechea, ${ }^{2}$ and Juan R. Pardo \\ Departamento de Astrofísica Molecular e Infrarroja, Instituto de Estructura de la Materia, CSIC, Serrano 121, 28006 Madrid, Spain
}

AND

AndrÉs AsEnsio-Ramos

Instituto de Astrofísica de Canarias, E-38205, La Laguna, Tenerife, Spain cerni@damir.iem.csic.es

Received 2005 November 18; accepted 2006 January 12

\begin{abstract}
Several condensations heated externally by nearby hot stars are present in the Sgr B2 region for which $\mathrm{H}_{2} \mathrm{O}$ far-IR lines are expected to probe only an external low-density and high temperature section. Millimeter-wave lines can penetrate deeper into them (higher densities and lower $T_{k}$ ). We have conducted a study combining $\mathrm{H}_{2} \mathrm{O}$ lines in both spectral regions using the $I S O$ (far-IR lines) and the IRAM $30 \mathrm{~m}$ telescope ( $183 \mathrm{GHz}$ line). The far-IR $\mathrm{H}_{2} \mathrm{O}$ lines, seen in absorption, are optically thick. They form in the outermost gas in front of the far-IR continuum sources, probing a maximum visual extinction of $\sim 5-10 \mathrm{mag}$. IR photons from the dust play a dominant role in their excitation. We conclude, based on observations of the $\mathrm{CO} J=7-6$ line at $806.65 \mathrm{GHz}$, and the lack of emission from the far-IR CO lines, that the gas density has to be below $\sim 10^{4} \mathrm{~cm}^{-3}$. Using the gas kinetic temperature and density derived from $\mathrm{OH}, \mathrm{CO}$, and other molecular species, we derive a water column density of $(9 \pm 3) \times 10^{16} \mathrm{~cm}^{-2}$ in the absorbing gas, implying an abundance of $\simeq(1-2) \times 10^{-5}$ in this region. The resulting relatively low $\mathrm{H}_{2} \mathrm{O} / \mathrm{OH}$ abundance ratio, $\simeq 2-4$, is a signature of UV photon-dominated surface layers traced by far-IR observations. As a consequence, the temperature of the absorbing gas is high, $T_{k} \simeq 300-500 \mathrm{~K}$, which allows very efficient neutral-neutral reactions producing $\mathrm{H}_{2} \mathrm{O}$ and $\mathrm{OH}$. Finally, the $183.31 \mathrm{GHz}$ data allow one to trace the inner, denser $\left(n\left(\mathrm{H}_{2}\right) \geq 10^{5}-10^{6} \mathrm{~cm}^{-3}\right)$, and colder $\left(T_{k} \sim 40 \mathrm{~K}\right)$ gas. The emission is very strong toward the cores with an estimated water vapor abundance of a few $\times 10^{-7}$. There is also moderate extended emission around Sgr B2 main condensations, in agreement with the water vapor abundance derived from far-IR $\mathrm{H}_{2} \mathrm{O}$ lines.
\end{abstract}

Subject headings: infrared: ISM — ISM: individual (Sgr B2) — ISM: lines and bands — ISM: molecules — radiative transfer

Online material: color figures

\section{INTRODUCTION}

The determination of water abundance in space is a longstanding problem in astronomy. Theoretical models predict that water can be the most abundant species in warm molecular clouds after $\mathrm{H}_{2}$ and $\mathrm{CO}$ (Neufeld et al. 1995). Therefore, the determination of its spatial distribution and abundance contributes to a better knowledge of the chemical and physical processes that take place in the interstellar medium (ISM).

Unfortunately, water is an abundant molecule in our atmosphere, making particularly difficult the observation of its rotational lines and vibrational bands from Earth. Even so, some observations of water lines have been performed from groundbased and airborne telescopes: the $6_{16}-5_{23}$ at $22 \mathrm{GHz}$ (Cheung et al. 1969), the $5_{15}-4_{22}$ at $325 \mathrm{GHz}$ (Menten et al. 1990b), the $10_{29}-9_{36}$ at $321 \mathrm{GHz}$ (Menten et al. 1990a), and the $3_{13}-2_{20}$ at $183.31 \mathrm{GHz}$ (Waters et al. 1980; Cernicharo et al. 1990, 1994, 1996, 1999; González-Alfonso et al. 1998). Due to the maser nature of these lines, the analysis and interpretation of the spectra is not obvious. Among these lines, only two have been used

\footnotetext{
${ }^{1}$ Based on observations with ISO, an ESA project with instruments funded by ESA Member States (especially the PI countries: France, Germany, the Netherlands, and the United Kingdom) and with participation of ISAS and NASA.

${ }^{2}$ Current address: Laboratoire d'Étude du Rayonnement et de la Matière, UMR 811, CNRS, Observatoire de Paris et École Normale Supérieure, 24 rue Lhomond, 75231 Paris Cedex 05, France.
}

to map the extended emission of water vapor in Orion: the $3_{13}-2_{20}$ (hereafter the $183 \mathrm{GHz}$ line; Cernicharo et al. 1994) and the $5_{15}-4_{22}$ at $325 \mathrm{GHz}$ (Cernicharo et al. 1999). However, although we know from Infrared Space Observatory (ISO) and Submillimeter Wave Astronomy Satellite (SWAS) observations that water is extended $\left(\sim 25^{\prime} \times 25^{\prime}\right)$ in Sgr B2 (Cernicharo et al. 1997; Neufeld et al. 2003; Goicoechea et al. 2004), little is known about its excitation conditions and its detailed spatial distribution. An alternative to indirectly estimate the water abundance in the Galactic center (GC) is to use related species such as HDO (Jacq et al. 1990; Comito et al. 2003) or $\mathrm{H}_{3} \mathrm{O}^{+}$(Phillips et al. 1992; Goicoechea \& Cernicharo 2001). In none of these cases is the determination of $\chi\left(\mathrm{H}_{2} \mathrm{O}\right)$ straightforward.

The ISO mission (Kessler et al. 1996) and, especially, the Long Wavelength Spectrometer (LWS; Clegg et al. 1996) and Short Wavelength Spectrometer (SWS; de Graauw et al. 1996) have provided a unique opportunity to observe several $\mathrm{H}_{2} \mathrm{O}$ lines in a great variety of astronomical environments. Nevertheless, the majority of these observations were performed at the low spectral resolution of the grating mode $\left(\sim 1000 \mathrm{~km} \mathrm{~s}^{-1}\right)$, which produces a critically strong dilution in the search for molecular features in most ISM sources. Nevertheless, the Sgr B2 cloud has been analyzed and studied in detail with the LWS FabryPérot (FP) interferometer, which provided a velocity resolution of $\sim 35 \mathrm{~km} \mathrm{~s}^{-1}$ (Goicoechea et al. 2004, hereafter G04).

Opposite to what is found toward other star-forming regions such as Orion (Cernicharo 1999), the observations of the 
$2_{12}-1_{01}$ line at $\sim 179.5 \mu \mathrm{m}$ in Sgr B2 show that the line appears in absorption rather than in emission (Cernicharo et al. 1997). Afterward, the launch of SWAS (Melnick et al. 2000) and ODIN (Nordh et al. 2003) allowed the observation of the $1_{10}-1_{01}$ fundamental transition of both $\mathrm{H}_{2}^{16} \mathrm{O}$ at 557 and $\mathrm{H}_{2}^{18} \mathrm{O}$ at $548 \mathrm{GHz}$, first detected by the Kuiper Airborne Observatory (Zmuidzinas et al. 1995). Although the velocity resolution is $\sim 1 \mathrm{~km} \mathrm{~s}^{-1}$, the large beam of $S W A S\left(\sim 4^{\prime}\right)$ makes these observations more sensitive to the cold and less dense gas. $S W A S$ observations have provided a reliable estimate of the water vapor abundance in the low-excitation clouds located in the line of sight toward Sgr B2 (Neufeld et al. 2000). However, the fact that only the ground-state absorption line is detected makes difficult a detailed study of water vapor excitation mechanisms in Sgr B2 itself. This is the most massive cloud in the Galaxy, with $\sim 10^{7} M_{\odot}$ (Lis \& Goldsmith 1990), and a paradigmatic object in the GC region as its geometrical properties, physical conditions, and chemical characteristics make it a miniature galactic nucleus with $\sim 15^{\prime}$ extent (G04 and references therein).

The main star-forming regions in Sgr B2 are located within three dust condensations, labeled (N), (M), and (S). These condensations are embedded in a $\sim 10 \mathrm{pc}$ moderate-density $\left[n\left(\mathrm{H}_{2}\right)=\right.$ $10^{5}-10^{6} \mathrm{~cm}^{-3}$ ] cloud (Lis \& Goldsmith 1991; Hüttemeister et al. 1993). In addition, these structures are surrounded by lower density components of warm $\left(T_{k} \geq 100 \mathrm{~K}\right)$ gas (hereafter the Sgr B2 warm envelope). These conditions have been derived mainly from absorption observations of $\mathrm{NH}_{3}$ metastable lines (Wilson et al. 1982; Hüttemeister et al. 1995), OH lines (Goicoechea \& Cernicharo 2001, 2002), and $\mathrm{H}_{2} \mathrm{CO}$ lines (Martín-Pintado et al. $1990)$ in the radio domain. Nevertheless, the warm and lowdensity gas is poorly traced by radio observations of other molecular species (generally excited by collisions in the denser regions and thus observed in emission). However, the warm envelope represents the strongest contribution to the absorption features produced by many light hydrides in the far-IR spectrum of Sgr B2 (G04). The origins of the observed rich chemistry and the heating mechanisms in the Sgr B2 warm envelope are a subject of intense debate. The matter is complicated due to the different observational signatures to be integrated in the same picture: high temperatures derived from $\mathrm{NH}_{3}$ absorption lines (Hüttemeister et al. 1995; Ceccarelli et al. 2002), fine structure emission from the photoionized and photodissociated gas (G04), $\mathrm{SiO}$ and X-ray distribution (Martín-Pintado et al. 2000), etc.

In all possible scenarios, water plays a significant role. Several mechanisms allow its formation and survival in the warm envelope. Dissociative recombination of $\mathrm{H}_{3} \mathrm{O}^{+}$leads to the production of $\mathrm{H}_{2} \mathrm{O}$ and $\mathrm{OH}$. These processes depend on the specific $f_{\mathrm{H}_{2} \mathrm{O}}$ branching ratio for the $\mathrm{H}_{2} \mathrm{O}$ formation channel. Unfortunately, the determination of $f_{\mathrm{H}_{2} \mathrm{O}}$ with different experimental procedures has also yielded different values, from $f_{\mathrm{H}_{2} \mathrm{O}}=0.05$ (Williams et al. 1996) to $f_{\mathrm{H}_{2} \mathrm{O}}=0.25$ (see Jensen et al. 2000), while most chemical models have used $f_{\mathrm{H}_{2} \mathrm{O}} \sim 0.35$. In addition, water could also be produced in the gas phase by the endothermic reaction:

$$
\mathrm{OH}+\mathrm{H}_{2} \rightarrow \mathrm{H}_{2} \mathrm{O}+\mathrm{H}
$$

However, the gas temperature must exceed $\sim 300 \mathrm{~K}$ to overcome the activation barrier (Neufeld et al. 1995). At these temperatures, the reaction

$$
\mathrm{O}+\mathrm{H}_{2} \rightarrow \mathrm{OH}+\mathrm{H}
$$

also contributes to the formation of $\mathrm{OH}$. Therefore, $\mathrm{H}_{2} \mathrm{O}$ and $\mathrm{OH}$ column densities can be used to determine the role of the neutralneutral reactions in their formation/destruction routes. Still, the exact $\mathrm{H}_{2} \mathrm{O} / \mathrm{OH}$ ratio will be determined by $f_{\mathrm{H}_{2} \mathrm{O}}$, the temperature, and by photodissociation processes if UV radiation is present. As an example, Neufeld et al. (2002) studied a diffuse cloud $\left[G_{0} \sim 1, n(\mathrm{H})=100 \mathrm{~cm}^{-3}\right]$ toward W51 and showed that the presence of a warm gas component $\left(T_{k} \gtrsim 400 \mathrm{~K}\right)$ could explain the observed variations of the $\mathrm{H}_{2} \mathrm{O} / \mathrm{OH}$ ratio with respect to other diffuse clouds.

Finally, high oxygen depletion onto water ice mantles in dust grains could have taken place during the evolution of the cool gas in Sgr B2. Photodesorption and/or evaporation for dust temperatures above $\sim 90 \mathrm{~K}$ could release some water back into the gas phase, enhancing the $\mathrm{H}_{2} \mathrm{O}$ abundance expected from pure gasphase formation (Bergin et al. 2000). However, gas and dust are thermally decoupled in the outer layers of Sgr B2, where the dust temperatures are significantly lower, $T_{d} \simeq 20-30 \mathrm{~K}$ (Gordon et al. 1993; G04) than gas temperatures, $T_{k} \simeq 300 \mathrm{~K}$ (Goicoechea $\&$ Cernicharo 2002; Ceccarelli et al. 2002). Thus, $T_{d}$ seems too low to produce significant evaporation of water ice mantles. Therefore, a detailed study of the far-IR $\mathrm{H}_{2} \mathrm{O}$ lines and the $183.31 \mathrm{GHz}$ extended emission is needed to constrain the water abundance and the physical characteristics of the absorbing/emitting region.

In this work we present and analyze the far-IR observations of several thermal lines of water vapor toward Sgr B2(M) and the first map of the $183.31 \mathrm{GHz}$ maser emission of para- $\mathrm{H}_{2} \mathrm{O}$ around Sgr B2 main condensations. The layout of the paper is as follows: In $\S 2$ we summarize the far-IR, submillimeter, and millimeter observations and data reduction. The spectra and maps are presented in $\S 3$. Section 4 is devoted to the analysis of CO observations ( $(4.1)$ and water vapor observations ( $(4.2)$ with different radiative transfer methods. The main implications of our work are discussed in $\S 5$, where photochemistry models for $\mathrm{H}_{2} \mathrm{O}$ and $\mathrm{OH}$ are also presented. A summary is given in $\S 6$.

\section{OBSERVATIONS AND DATA REDUCTION}

\subsection{Far-IR Observations}

Most pure rotational lines of water vapor that play a role in the radiative heating and cooling of the dense ISM appear in the terahertz domain. Thus, airborne or satellite observations are needed to avoid the Earth's atmosphere blocking. In particular, many pure rotational lines of $\mathrm{H}_{2} \mathrm{O}$ appear in the far-IR coverage of the LWS spectrometer (Clegg et al. 1996) on board ISO (Kessler et al. 1996). We have used the LWS-FP instrument to search for $\mathrm{H}_{2} \mathrm{O}$ and $\mathrm{H}_{2}^{18} \mathrm{O}$ lines toward Sgr B2(M). The LWS-FP spectral resolution is $\lambda / \Delta \lambda \simeq 7000-1000$ and has a circular aperture of about $80^{\prime \prime}$ in diameter. The majority of detected lines have been observed in the time awarded to our ISO proposals. However, an extensive inspection of the public ISO database ${ }^{3}$ has been carried out in order to examine and average all available water lines. Most of the water lines were present in the Astronomical Observation Template (AOT) LWS04 observations, which give a large spectral sampling, wavelength precision, and signal-to-noise ratios. The number of scans in this mode was $\geq 12$, depending on the expected absorption produced by the different species.

These LWS products have been processed and compared through the off-line-processing (OLP) pipeline, versions 6.0 and

\footnotetext{
3 The ISO database observations (TDTs) presented in these work are 32201428 , 32201429, 46201118, 46201123, 46900332, 47600907, 47600908, 47600909, 47601001 , and 49800301 .
} 
10.1. There are no major differences except that recent pipelines produce $<10 \%$ less absorption in some lines due to continuum level differences from one OLP to another. The data were analyzed using the ISO spectrometer data reduction package ISAP. ${ }^{4}$ The mean FP continuum flux of each line deviates by $<20 \%$, and this can be taken as the flux calibration error (Swinyard et al. 1998; Gry et al. 2003). After checking the continuum level of the AOT LWS01 observations, a polynomial baseline was fitted to each spectra and adopted as the FP continuum level.

\subsection{Millimeter and Submillimeter Observations}

The water line at $183.31 \mathrm{GHz}$ can be observed from high mountain-top sites under very dry conditions (Cernicharo et al. 1990). The observations in the direction of Sgr B2 were achieved with the IRAM $30 \mathrm{~m}$ telescope, with a half-power beamwidth (HPBW) of $\sim 13^{\prime \prime}$, in 1999 December and 2004 January. The source transits at $24^{\circ}$ elevation at Pico Veleta, but even so the average atmospheric opacity over a bandwidth of $0.5 \mathrm{GHz}$ centered at $183.3 \mathrm{GHz}$ was 1.7 , i.e., an atmospheric water column of $0.9 \mathrm{~mm}$ along the line of sight. We used an SIS receiver designed to cover the band 130-184 GHz. In its higher end, the receiver temperature is about $160 \mathrm{~K}$, and the sideband rejection is at least $18 \mathrm{~dB}$. The back end used was a 512 two-pole filter with halfpower widths and spacings equal to $1.0 \mathrm{MHz}$. With these conditions, and in this configuration, system temperatures were typically around $3000 \mathrm{~K}$. The pointing was checked using the already known strong emission of W49N (Cernicharo et al. 1990). Once it was verified that the Sgr B2 emission was quite compact, the map was carried out in wobbler-switching mode in order to obtain very flat baselines. Observations were also tried in 2001 January, 2002 January, and 2004 January. In the 2001 run the weather was poor, while in the last run it was possible to have a 30 minute window of observing time with good atmospheric transmission. These runs allowed us to check that the line profile in the position peak of Sgr B2(M) was the same as 1999 December and to confirm that the extent of the water emission toward this condensation was larger than the beam size. In the 2004 January observing run we also had very good atmospheric transmission to perform observations around Sgr B2(N). However, the measured line fluxes were lower than those in previous observations by a constant factor. As the line profiles did not change, we scaled the 1999 observations to those of the reference taken in the $\left(0^{\prime \prime},+5^{\prime \prime}\right)$ position in 2004 (see Fig. 2).

The CO 7-6 (806 GHz) observations were performed on 2002 March 10 with the $10.4 \mathrm{~m}$ Caltech Submillimeter Observatory (CSO) located at the summit of Mauna Kea (Hawaii). The receiver is a helium-cooled SIS mixer operating in double-sideband mode (DSB), providing an instantaneous bandwidth of $0.95 \mathrm{GHz}$ and designed to fully cover the 780-910 GHz atmospheric window (see Kooi et al. 2000). The pointing was checked using a strong CO pointlike source in the nearby W28 molecular cloud and was kept within $3^{\prime \prime}$ accuracy for a HPBW of $\sim 10^{\prime \prime}$. Two different acousto-optic spectrometer back ends were used with 2048 and 1024 channels, respectively, for a total spectral coverage of 1.5 and $0.5 \mathrm{GHz}$ in each case. The zenith atmospheric water vapor column was $\sim 0.5 \mathrm{~mm}$ during the observations, resulting in system temperatures ranging from 6000 to $10,000 \mathrm{~K}$ depending on the receiver and the elevation. $\mathrm{CO}$ emission is very extended around Sgr B2, so we had to perform position-switched scans setting the

\footnotetext{
4 ISAP is a joint development by the LWS and SWS Instrument Teams and Data Centers. Contributing institutes are CESR, IAS, IPAC, MPE, RAL, and SRON.
}

off position $1^{\circ}$ away in azimuth. An additional 5\% increase had to be applied to $T_{A}^{*}$ in order to second-order correct the standard "chopper-wheel" calibration method at high frequencies with large atmospheric opacities (see Pardo et al. 2005).

\section{RESULTS}

The far-IR spectrum of Sgr B2(M) is dominated by the absorption produced by $\mathrm{NH}_{3}, \mathrm{OH}$, and $\mathrm{H}_{2} \mathrm{O}$ rotational lines (G04). The detected far-IR lines of water vapor are shown in Figure 1. $\mathrm{H}_{2} \mathrm{O}$ spectroscopical and observational data are tabulated in Table 1. Except for the $2{ }_{12}-1_{01}$ ground state line of $\mathrm{o}-\mathrm{H}_{2} \mathrm{O}$ at $\sim 179.5 \mu \mathrm{m}(\sim 1669.9 \mathrm{GHz})$, all water lines have a similar profile and are centered at Sgr B2(M) velocities. The $\sim 179.5 \mu \mathrm{m}$ line is saturated and absorbs from -150 to $+100 \mathrm{~km} \mathrm{~s}^{-1}$, which therefore includes the water vapor located in the foreground gas toward the GC and the warm gas around Sgr B2(M). The widespread absorption produced by the $2_{12}-1_{01}$ line has been previously presented in Cernicharo et al. (1997) and enlarged in G04 $\left(9^{\prime} \times 27^{\prime}\right)$, while the $1_{10}-1_{01}$ absorption has been mapped $\left(26^{\prime} \times 19^{\prime}\right)$ by $S W A S$ (Neufeld et al. 2003). These observations prove that low-excitation $\mathrm{H}_{2} \mathrm{O}$ is present in the clouds intersecting the line of sight toward large areas of Sgr B2.

The average velocity of all $\mathrm{H}_{2} \mathrm{O}$ lines observed with the $I S O$ LWS-FP is $+60 \pm 5 \mathrm{~km} \mathrm{~s}^{-1}$, in agreement with the velocity of other related oxygen species such as $\mathrm{H}_{3} \mathrm{O}^{+}$or $\mathrm{OH}$ (Goicoechea $\&$ Cernicharo 2001, 2002). Taking into account the wavelength calibration error of the LWS-FP instrument, this velocity is compatible with the expected $+65 \mathrm{~km} \mathrm{~s}^{-1}$ cloud seen in radio observations (Hüttemeister et al. 1995). We note, however, that velocities close to $+60 \mathrm{~km} \mathrm{~s}^{-1}$ are also associated with gas surrounding most Sgr B2 continuum sources, so that the bulk of the $\mathrm{H}_{2} \mathrm{O}$ absorption can arise from them.

Possible overlapping with other molecular species occurs at some wavelengths. In particular, the $\mathrm{o}-\mathrm{H}_{2} \mathrm{O} 4_{32}-4_{23}$ line is blended with HF $J=2-1$ at $121.697 \mu \mathrm{m}$ (Neufeld et al. 1997) and the $\mathrm{o}-\mathrm{H}_{2}^{18} \mathrm{O} 2_{12}-1_{01}$ line at $181.053 \mu \mathrm{m}$ has a small contribution from $\mathrm{H}_{3} \mathrm{O}^{+} Q(1,1)$ (Goicoechea \& Cernicharo 2001).

Observations of the $183.31 \mathrm{GHz} \mathrm{H} \mathrm{H}_{2} \mathrm{O}$ line $\left(E_{u} \simeq 205 \mathrm{~K}\right)$ toward Sgr B2 are presented in Figure 2. The emission appears at the LSR velocities of Sgr B2 with no contribution from the line-of-sight clouds. The emission appears at least in the 50$75 \mathrm{~km} \mathrm{~s}^{-1}$ range. Note the different line shapes and intensities of the $183.31 \mathrm{GHz}$ emission for positions in front and around the main condensations (here the line appears much wider).

Finally, Figure 3 shows the CO $J=7-6$ line $\left(E_{u} \simeq 150 \mathrm{~K}\right)$ observed toward Sgr B2(M). This CO line shows a strong selfabsorption at $\sim 70 \mathrm{~km} \mathrm{~s}^{-1}$ (the velocity of the $183.31 \mathrm{GHz} \mathrm{H}_{2} \mathrm{O}$ line peak at his position) so that the profile peaks at $\sim+55$ and $+85 \mathrm{~km} \mathrm{~s}^{-1}$. Similar patterns are shown by lower- $J \mathrm{CO}$ lines.

\section{ANALYSIS AND DISCUSSION}

\subsection{Carbon Monoxide}

The warm gas present in the outer layers of Sgr B2(M) might be expected to radiate in high- $J$ CO lines. The CO $J=14-13$ transition at $185.999 \mu \mathrm{m}$ is the one with the lowest energy level $\left(E_{u} \simeq 581 \mathrm{~K}\right)$ within the range of ISO LWS detectors. However, we have not detected any emission/absorption from $\mathrm{CO}$ (3 $\sigma$ limits are $<2 \times 10^{-18} \mathrm{~W} \mathrm{~cm}^{-2}$ ) in the ISO LWS spectra toward the Sgr B2 region (G04), with both grating and FP. CO spectroscopical data and line flux upper limits are tabulated in Table 2. Nevertheless, recent studies of the large-scale $\mathrm{CO} J=7-6$ emission toward the GC with the Antarctic Submillimeter Telescope and 


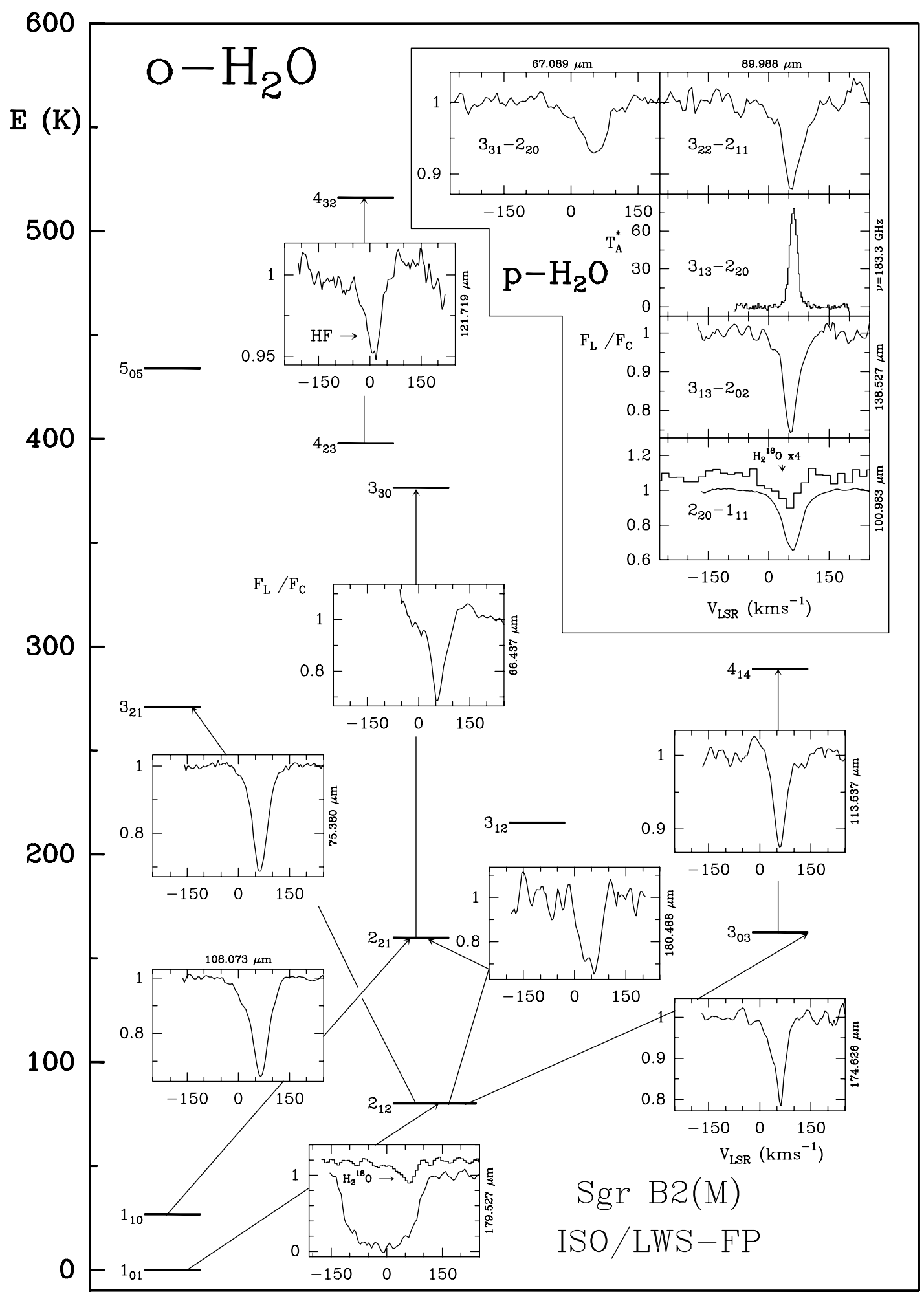

FIg. 1.- ISO LWS-FP observations of $\mathrm{H}_{2}^{16} \mathrm{O}$ and $\mathrm{H}_{2}^{18} \mathrm{O}$ toward $\mathrm{Sgr} \mathrm{B} 2(\mathrm{M})$ and rotational energy diagram of ortho- $\mathrm{H}_{2}^{16} \mathrm{O}$. The ordinate scale corresponds to $F / F_{c}$, and the abscissa to the wavelength in microns. All the far-IR water lines appear in absorption. The emission line corresponds to the $3_{13}-2_{20}$ maser transition of para$\mathrm{H}_{2}^{16} \mathrm{O}$ at $183.31 \mathrm{GHz}$ (observed with the IRAM $30 \mathrm{~m}$ telescope at Pico Veleta, Spain). [See the electronic edition of the Journal for a color version of this figure.]

Remote Observatory (AST/RO) have shown that the emission is concentrated toward Sgr B and Sgr A complexes (Kim et al. 2002). Figure 3 shows higher spectral/angular resolution observations of this line taken with the CSO telescope toward the Sgr B2(M) position. Hence, it seems that at a given $J_{\text {up }}$ level, the rotational $\mathrm{CO}$ line emission disappears from the $\mathrm{Sgr} \mathrm{B} 2$ spectrum.
We have performed nonlocal radiative transfer calculations to try to reproduce the lack of high- $J \mathrm{CO}$ lines in the far-IR spectrum of Sgr B2(M) and to help constrain the physical parameters needed to model the water vapor absorption. In Figure 4 we show the predictions of a nonlocal model for several high- $J$ transitions of $\mathrm{CO}$. The nonlocal model used here is an adaptation 
TABLE 1

Summary of $\mathrm{H}_{2} \mathrm{O}$ Spectroscopical and Observational Data Presented in This Work

\begin{tabular}{|c|c|c|c|c|c|c|c|c|}
\hline Species & Transition & $\begin{array}{c}\lambda \\
(\mu \mathrm{m})\end{array}$ & $\begin{array}{c}\nu \\
(\mathrm{GHz})\end{array}$ & $\begin{array}{c}E_{\text {upper }} \\
(\mathrm{K})\end{array}$ & $\begin{array}{c}A_{i j} \\
\left(\mathrm{~s}^{-1}\right)\end{array}$ & $\begin{array}{c}F_{l} \\
\left(\mathrm{~W} \mathrm{~cm}^{-2}\right)\end{array}$ & $F / F_{c}$ & $\begin{array}{c}\Delta v \\
\left(\mathrm{~km} \mathrm{~s}^{-1}\right)\end{array}$ \\
\hline $\mathrm{p}-\mathrm{H}_{2} \mathrm{O}$ & $3_{13}-2_{20}$ & 1635.439 & 183.3 & 205 & $3.53 \mathrm{E}-06$ & $1120 \pm 60 \mathrm{~K} \mathrm{~km} \mathrm{~s}^{-1}$ & & $30 \pm 3$ \\
\hline $\mathrm{o}-\mathrm{H}_{2} \mathrm{O}$ & $2_{21}-2_{12}$ & 180.488 & 1661.0 & 160 & $2.99 \mathrm{E}-02$ & $-(3.44 \pm 0.46) \mathrm{E}-18$ & $0.74 \pm 0.01$ & $64 \pm 3$ \\
\hline $\mathrm{o}-\mathrm{H}_{2} \mathrm{O}$ & $2_{12}-1_{01}$ & 179.527 & 1669.9 & 80 & $5.47 \mathrm{E}-02$ & $-(6.76 \pm 0.29) \mathrm{E}-14$ & $0.06 \pm 0.03$ & $204 \pm 23$ \\
\hline $\mathrm{o}-\mathrm{H}_{2} \mathrm{O}$ & $3_{03}-2_{12}$ & 174.626 & 1716.7 & 163 & $4.94 \mathrm{E}-02$ & $-(1.86 \pm 0.46) \mathrm{E}-18$ & $0.82 \pm 0.02$ & $44 \pm 2$ \\
\hline $\mathrm{p}-\mathrm{H}_{2} \mathrm{O}$ & $3_{13}-2_{02}$ & 138.527 & 2164.1 & 205 & $1.22 \mathrm{E}-01$ & $-(1.27 \pm 0.03) \mathrm{E}-18$ & $0.89 \pm 0.02$ & $41 \pm 2$ \\
\hline $\mathrm{p}-\mathrm{H}_{2}^{18} \mathrm{O}$ & $2_{20}-1_{11}$ & 102.008 & 2938.9 & 194 & $2.52 \mathrm{E}-01$ & $-(9.38 \pm 2.62) \mathrm{E}-19$ & $0.96 \pm 0.01$ & $72 \pm 8$ \\
\hline $\mathrm{p}-\mathrm{H}_{2} \mathrm{O}$ & $2_{20}-1_{11}$ & 100.983 & 2968.7 & 196 & $2.55 \mathrm{E}-01$ & $-(6.02 \pm 0.11) \mathrm{E}-18$ & $0.66 \pm 0.01$ & $55 \pm 9$ \\
\hline $\mathrm{p}-\mathrm{H}_{2} \mathrm{O}$ & $3_{22}-2_{11}$ & 89.988 & 3331.4 & 297 & $3.45 \mathrm{E}-01$ & $-(1.99 \pm 0.14) \mathrm{E}-18$ & $0.90 \pm 0.01$ & $67 \pm 2$ \\
\hline $\mathrm{o}-\mathrm{H}_{2} \mathrm{O}$ & $3_{21}-2_{12}$ & 75.380 & 3977.0 & 271 & $3.25 \mathrm{E}-01$ & $-(4.32 \pm 0.07) \mathrm{E}-18$ & $0.69 \pm 0.02$ & $51 \pm 2$ \\
\hline $\mathrm{p}-\mathrm{H}_{2} \mathrm{O}$ & $3_{31}-2_{20}$ & 67.089 & 4468.6 & 410 & $1.20 \mathrm{E}+00$ & $-(1.36 \pm 0.08) \mathrm{E}-18$ & $0.93 \pm 0.03$ & $76 \pm 8$ \\
\hline $\mathrm{o}-\mathrm{H}_{2} \mathrm{O}$ & $3_{30}-2_{21}$ & 66.437 & 4512.4 & 376 & $1.22 \mathrm{E}+00$ & $-(8.39 \pm 0.14) \mathrm{E}-19$ & $0.92 \pm 0.02$ & $46 \pm 3$ \\
\hline
\end{tabular}

Notes.-Uncertainties refer to fitting errors. The values provided for the $183 \mathrm{GHz}$ line correspond to the direction of Sgr B2( M). In this case, the area of the observed line in $\mathrm{K} \mathrm{km} \mathrm{s}^{-1}$ is provided instead of the flux. Note that energy levels take into account that both ortho and para ground states are at $0 \mathrm{~K}$.

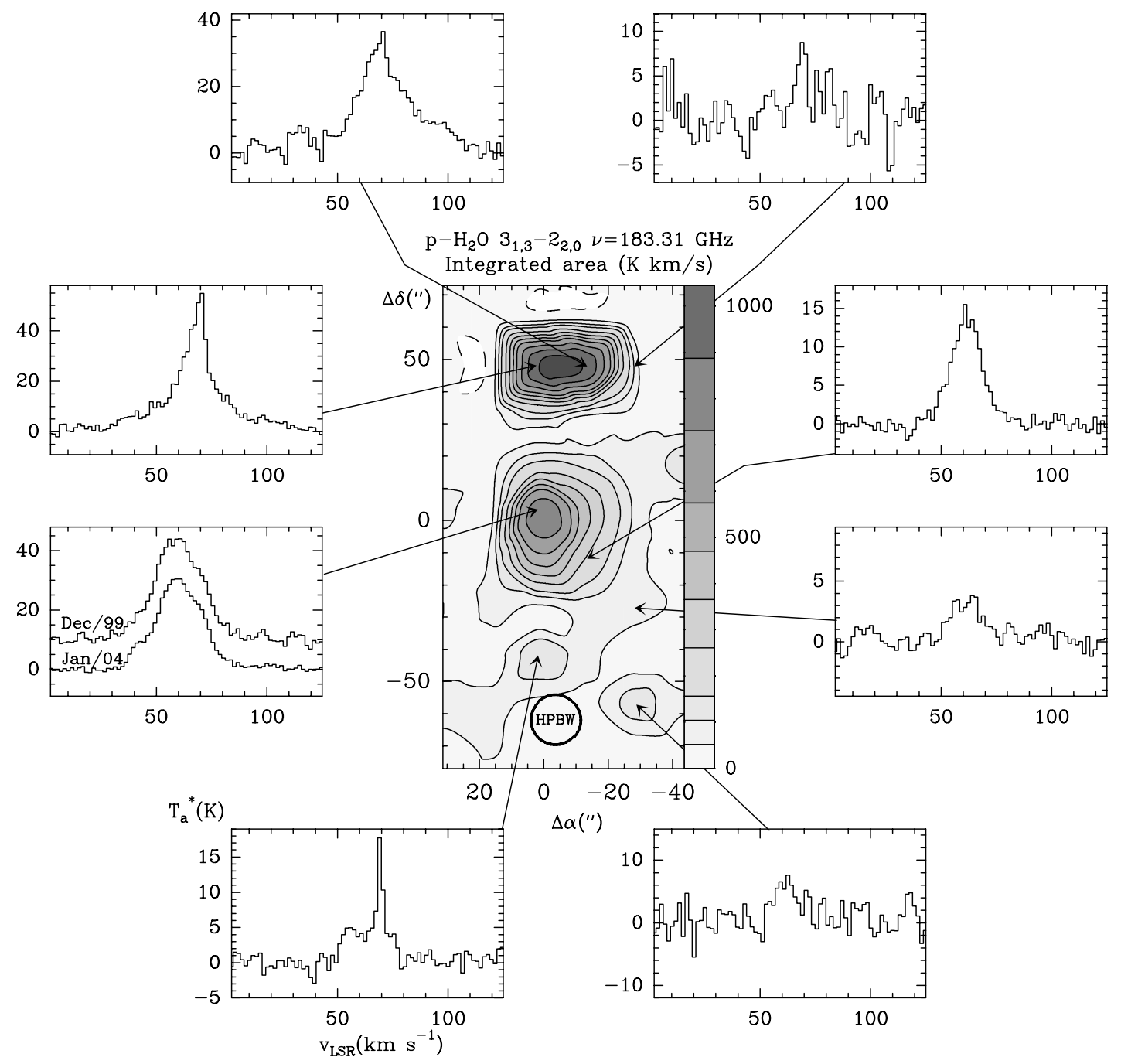

Fig. 2. - Map of the $3_{13}-2_{20}$ line of para- $\mathrm{H}_{2}^{16} \mathrm{O}$ at $183.31 \mathrm{GHz}$ around $\mathrm{Sgr} \mathrm{B} 2$ main condensations. Contours in $\mathrm{K} \mathrm{km} \mathrm{s}{ }^{-1}$ are indicated in the figure. Different line profiles at significant positions of the map are also shown. The intensity scale is in $T_{A}^{*}$ and the abscissa is the LSR velocity in km ${ }^{-1}$. The map is centered at the position of Sgr B2(M). The spectra were obtained in two different runs in 1999 December and 2004 January. The fluxes were scaled using the $\left(0^{\prime \prime}, 5^{\prime \prime}\right)$ position as reference. Note that the line profiles in that reference position did not change in more than $4 \mathrm{yr}$. 


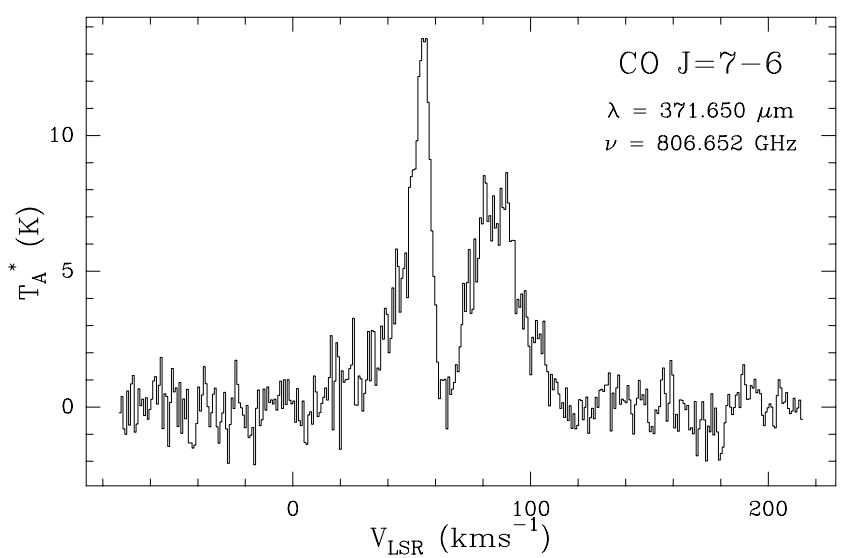

FIG. 3.-CSO observation of the $\mathrm{CO} J=7-6$ line at $806 \mathrm{GHz}$ toward Sgr B2(M).

of the radiative transfer code developed by González-Alfonso \& Cernicharo (1993) with the inclusion of dust in the transfer (Cernicharo et al. 2000). We have implemented it for high- $J$ levels of $\mathrm{CO}$ [see $\S$ 4.2.2 for further details on the specific model developed for Sgr B2(M)]. The collisional rates have been taken from Flower (2001). The dust continuum emission has been modeled following Goicoechea \& Cernicharo (2002) and G04 (see $\S$ 4.2.1). We have run an array of models for $\mathrm{CO}$ abundances ${ }^{5}$ of $10^{-4}$ and $10^{-5}$ with $T_{k}$ varied from 100 to $500 \mathrm{~K}$ and $n\left(\mathrm{H}_{2}\right)$ from $5.0 \times 10^{3}$ to $6.4 \times 10^{5} \mathrm{~cm}^{-3}$. From these results it is clear that in order to suppress the far-IR CO emission and to match the $\mathrm{CO} J=7-6$ line emission, low- $\mathrm{H}_{2}$ density is required. In particular, if the $\mathrm{CO} J=7-6$ line arises from a layer of gas at $T_{k} \sim 100 \mathrm{~K}$, the model with $n\left(\mathrm{H}_{2}\right)=2 \times 10^{4} \mathrm{~cm}^{-3}$ correctly reproduces the absence of far-IR lines. However, if it arises from the same outer layer of warm $\mathrm{OH}\left(T_{k} \sim 300 \mathrm{~K}\right)$ detected in the far-IR (Goicoechea \& Cernicharo 2002), the limit for the density will be $<10^{4} \mathrm{~cm}^{-3}$. In any case, the lack of high- $J$ CO lines is evidence of the low-density gas located in front of the far-IR continuum source. In the following section we constrain the physical parameters of this layer by studying the available water vapor lines.

\subsection{Water Vapor}

The main observational result of this work is that the far-IR water lines toward Sgr B2(M) appear in absorption, while the $183.31 \mathrm{GHz}$ line is seen in emission in and around the main condensations. The fact that the continuum emission in the far-

\footnotetext{
5 An upper limit to the ${ }^{13} \mathrm{CO}$ fractional abundance in Sgr B2 of $10^{-6}$ was found by Lis \& Goldsmith (1989). According to ${ }^{12} \mathrm{C}^{18} \mathrm{O} /{ }^{13} \mathrm{C}^{18} \mathrm{O} \simeq 25$ in Sgr B2 (Langer \& Penzias 1990 ), the ${ }^{12} \mathrm{CO}$ abundance would be $\lesssim 2.5 \times 10^{-5}$.
}

IR is optically thick (G04) indicates that the $\mathrm{H}_{2} \mathrm{O}$ absorption lines arise from regions where the excitation temperatures $\left(T_{\mathrm{ex}}\right)$ are smaller than the dust temperatures inferred from the continuum emission. However, the problem of the $\mathrm{H}_{2} \mathrm{O}$ line excitation toward Sgr B2 is not straightforward. Apart from self-absorption and the possible excitation by collisions with molecules (e.g., $\mathrm{H}_{2}$ ), atoms (e.g., He), and $e^{-}$(if the ionization fraction is significant), the level population can be primarily determined by the thermal emission of dust, the role of which is essential in the excitation of molecules such as $\mathrm{H}_{2} \mathrm{O}$ or $\mathrm{OH}$, which have many rotational lines in the far- and mid-IR. This represents a major difference with respect to the excitation treatment of molecules observed in the radio domain where, generally, one can neglect the excitation by dust photons. If the excitation is dominated by IR photons, the two transitions arising from the ground levels of orthowater, $2_{12}-1_{01}$ and $1_{10}-1_{01}$, will determine how the higher energy levels will be populated. Even if collisions are important, the presence of an optically thick far-IR continuum will strongly affect the $T_{\mathrm{ex}}$ of water, and thus it must be carefully taken into account in the models. In the case of Sgr B2, this means that the external dust layers of the cloud will absorb the possible water line emission from the inner regions. Knowledge of the geometry of the region to be modeled and the relative filling factors of the dust and gas in the beam of the LWS instrument are also important for the models. For this reason, high angular resolution ground-based observations of the $183.31 \mathrm{GHz}$ water line are particularly important to model the water vapor radiative transfer in Sgr B2.

\subsubsection{Large Velocity Gradient Modeling}

In this section we analyze the $\mathrm{H}_{2} \mathrm{O}$ observations with a multimolecule large velocity gradient (LVG) model. For Sgr B2(M) we have adopted a spherical geometry with two components (see Fig. 5): a uniform continuum core with a diameter of $\sim 23^{\prime \prime}(\sim 1 \mathrm{pc}$ for a distance of $8.5 \mathrm{kpc}$ ) and a shell of variable thickness and distance to the core. The presence of an external shell of molecular gas (not resolved by the ISO LWS beam) surrounding a central condensation is indicated by the analysis of the far-IR $\mathrm{OH}$ (Goicoechea \& Cernicharo 2002) and $\mathrm{NH}_{3}$ lines (Ceccarelli et al. 2002), and it is also supported by $\mathrm{H}_{2} \mathrm{O} 183.31 \mathrm{GHz}$ line observations (Fig. 2). In particular, Goicoechea \& Cernicharo (2002) found an angular size of $\sim 42^{\prime \prime}$ for the $\mathrm{OH}$ envelope. The bulk of the $\mathrm{H}_{2} \mathrm{O}$ absorption lines must arise from the $\mathrm{OH}$ layers or inside them. Following the $\mathrm{OH}$ model geometry and Figures 2 and 3, a total size of $\sim 30^{\prime \prime}(\sim 1.2 \mathrm{pc})$ for the core+shell cloud has been adopted. The core is considered as a graybody with an opacity at $80 \mu \mathrm{m}$ of 2.5 , with a dust opacity law given by $\tau_{\lambda}=$ $\tau_{80}[80 / \lambda(\mu \mathrm{m})]$, and a dust temperature of $30 \mathrm{~K}$. These values are consistent with the color temperatures and dust emissivities derived from the analysis of the ISO LWS continuum observations at the same wavelengths of the detected far- $\mathrm{IR}_{2} \mathrm{O}$ lines

TABLE 2

Summary of Far-IR CO Spectroscopic and Observational Data Presented in This Work

\begin{tabular}{|c|c|c|c|c|c|c|c|}
\hline Species & Transition & $\begin{array}{c}\lambda \\
(\mu \mathrm{m})\end{array}$ & $\begin{array}{c}\nu \\
(\mathrm{GHz})\end{array}$ & $\begin{array}{c}E_{\text {upper }} \\
(\mathrm{K})\end{array}$ & $\begin{array}{c}A_{i j} \\
\left(\mathrm{~s}^{-1}\right)\end{array}$ & $\begin{array}{c}F_{l} \\
\left(\mathrm{~W} \mathrm{~cm} \mathrm{~cm}^{-2}\right)\end{array}$ & $F / F_{c}$ \\
\hline $\mathrm{CO} \ldots$ & $14-13$ & 185.9 & 1611.8 & 581 & $2.95 \mathrm{E}-04$ & $<2.28 \mathrm{E}-18$ & $<1.007$ \\
\hline $\mathrm{CO} \ldots \ldots \ldots \ldots$ & $15-14$ & 173.6 & 1726.6 & 663 & $3.64 \mathrm{E}-04$ & $<1.02 \mathrm{E}-18$ & $<1.003$ \\
\hline $\mathrm{CO} \ldots \ldots \ldots \ldots \ldots \ldots \ldots$ & $16-15$ & 162.8 & 1841.3 & 752 & $4.42 \mathrm{E}-04$ & $<1.14 \mathrm{E}-18$ & $<1.003$ \\
\hline $\mathrm{CO} \ldots \ldots \ldots \ldots \ldots \ldots$ & $17-16$ & 153.3 & 1956.0 & 846 & $5.31 \mathrm{E}-04$ & $<1.49 \mathrm{E}-18$ & $<1.003$ \\
\hline
\end{tabular}

Notes.-No far-IR CO line has been detected. $3 \sigma$ upper limit line fluxes are tabulated. 


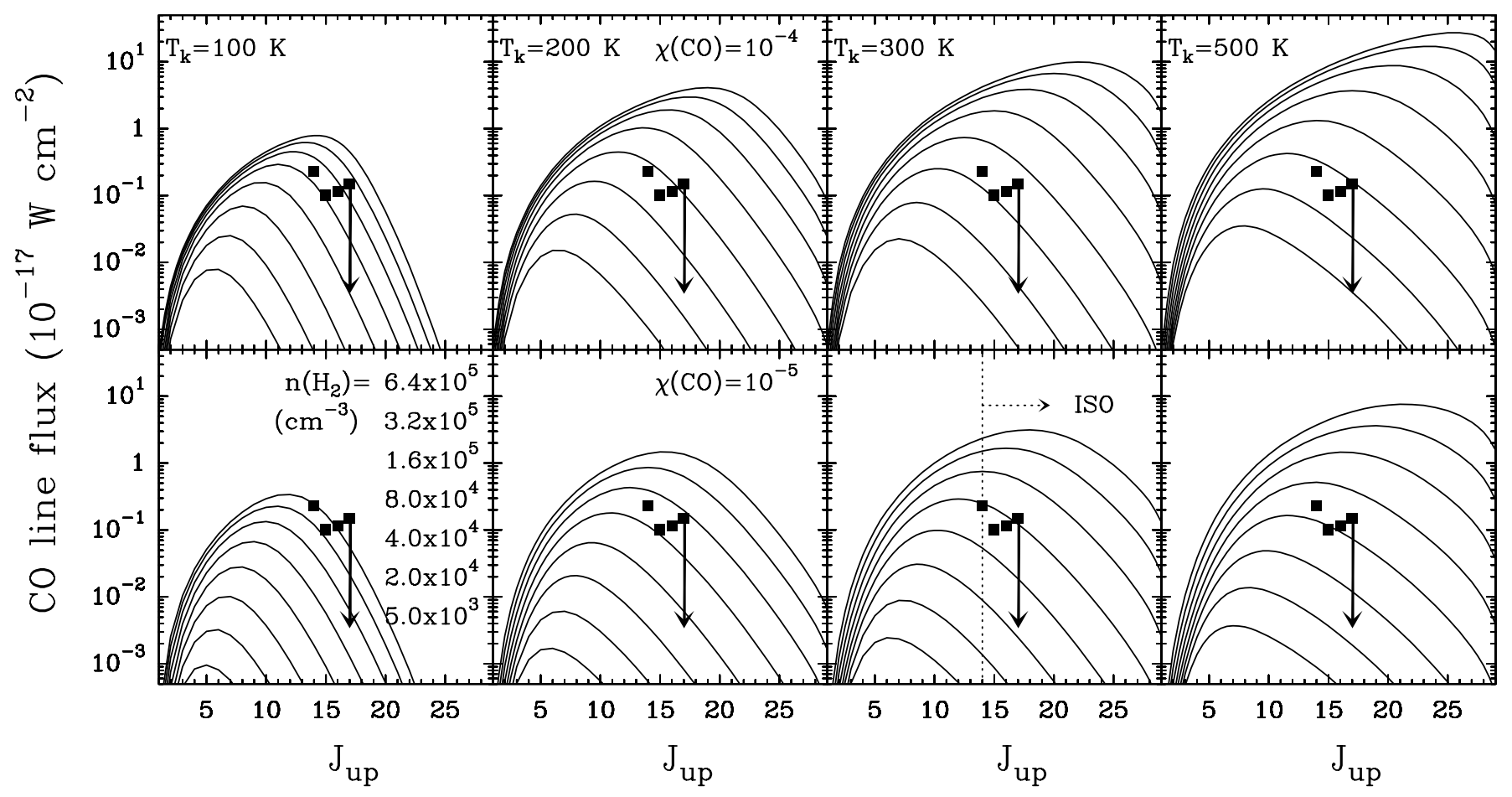

FIG. 4.-Nonlocal models of CO rotational lines toward Sgr B2. Velocity-integrated line fluxes (in $10^{-17} \mathrm{~W} \mathrm{~cm}^{-2}$ ) are shown as a function of $J_{\text {up }}$, the CO upper level rotational number. From $J=14-13$, CO lines are accessible to the ISO LWS (vertical dashed line); however, no CO line has been detected. $3 \sigma$ upper limits for CO line fluxes are shown ( filled squares). Two different $\mathrm{CO}$ abundances have been considered, $\chi(\mathrm{CO})=10^{-4}$ (top panels) and $\chi(\mathrm{CO})=10^{-5}$ (bottom panels). Models with different kinetic temperatures $(100-500 \mathrm{~K})$ are shown in each panel for different $\mathrm{H}_{2}$ volume densities: $5.0 \times 10^{3}, 1.0 \times 10^{4}, 2.0 \times 10^{4}, 4.0 \times 10^{4}$, $8.0 \times 10^{4}, 1.6 \times 10^{5}, 3.2 \times 10^{5}, 3.2 \times 10^{5}$, and $6.4 \times 10^{5} \mathrm{~cm}^{-3}$. [See the electronic edition of the Journal for a color version of this figure.]

(G04). Although dust temperatures can be slightly larger $\left(T_{d} \sim\right.$ $60-80 \mathrm{~K}$ ) inside the cloud (from the analysis of the millimeter continuum emission) or slightly lower $\left(T_{d} \sim 20 \mathrm{~K}\right)$ in the outer and colder diffuse layers (from the analysis of extended IRAS continuum emission; Gordon et al. 1993), we judged $T_{d} \simeq 30 \mathrm{~K}$ as the most representative value for the dust grains coexisting with the molecular species detected in the far-IR. The bulk of the continuum emission detected by ISO can hardly be fitted with dust temperatures below $30 \mathrm{~K}$. The emission of higher temper-

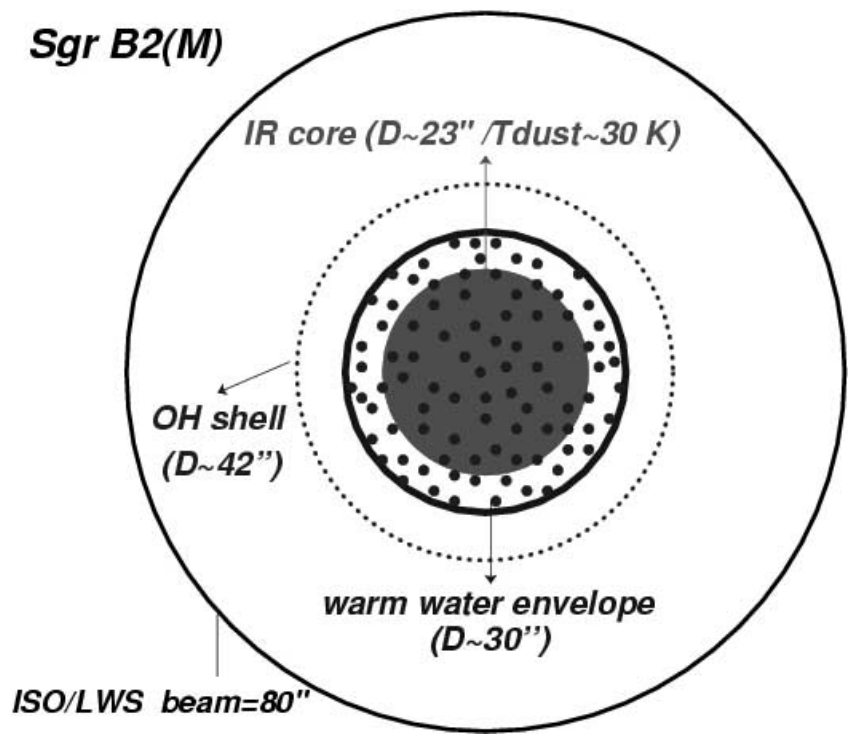

FIG. 5.- Sketch of the water model for Sgr B2(M). The dimensions of the $\mathrm{OH}$ envelope are also shown (Goicoechea \& Cernicharo 2002). [See the electronic edition of the Journal for a color version of this figure.] ature dust arising from the innermost regions of Sgr B2 is hidden in the FIR by the huge amount of foreground gas and colder dust.

For these models we have considered an ortho- $\mathrm{H}_{2} \mathrm{O}$ column density of $1.8 \times 10^{16} \mathrm{~cm}^{-2}$ and a para- $\mathrm{H}_{2} \mathrm{O}$ column density of $0.6 \times 10^{16} \mathrm{~cm}^{-2}$. These are lower limits suggested by the nonlocal radiative transfer models (see below). The LVG model computes the statistical equilibrium population of the rotational levels for ortho- $\mathrm{H}_{2} \mathrm{O}$ and para- $\mathrm{H}_{2} \mathrm{O}$ independently. The collisional rates were scaled from those of $\mathrm{H}_{2} \mathrm{O}-\mathrm{He}$ collisions (Green et al. 1993).

Several LVG computations for some selected ortho- and para$\mathrm{H}_{2} \mathrm{O}$ transitions in the terahertz domain are shown in Figure 6. The excitation temperature $T_{\mathrm{ex}}$ of each transition is shown in each panel as a function of $T_{k}$ and $n\left(\mathrm{H}_{2}\right)$. The thick contour corresponds to the equivalent temperature of the continuum core. Therefore, to the left of this contour, water lines appear in absorption. From the LVG models it is clear that high density and temperature are required to observe the far-IR water lines in emission. For the range of densities implied by the $\mathrm{CO}$ observations $\left(<10^{4} \mathrm{~cm}^{-3}\right)$, the $\mathrm{H}_{2} \mathrm{O}$ lines observed by the ISO LWS are correctly predicted in absorption. The temperature of the absorbing layer is, however, more difficult to estimate because the possible solutions for a given (low) density model are not very sensitive to temperature variations, as indicated by the smooth change of $T_{\mathrm{ex}}$ for constant density as the kinetic temperature goes from 500 to $100 \mathrm{~K}$. LVG models predict that, in addition to dust photons, collisions play a role in the excitation of the lowest $\mathrm{H}_{2} \mathrm{O}$ rotational levels. The higher energy levels are pumped from the lowest levels by absorption of far-IR photons. In the case of the ortho- $\mathrm{H}_{2} \mathrm{O} 1_{10}-1_{01}$ line observed by $S W A S$ (Neufeld et al. 2000), only moderate densities $\left(>5 \times 10^{4} \mathrm{~cm}^{-3}\right)$ are needed to observe the line in emission. This is the case of the extended emission in the 


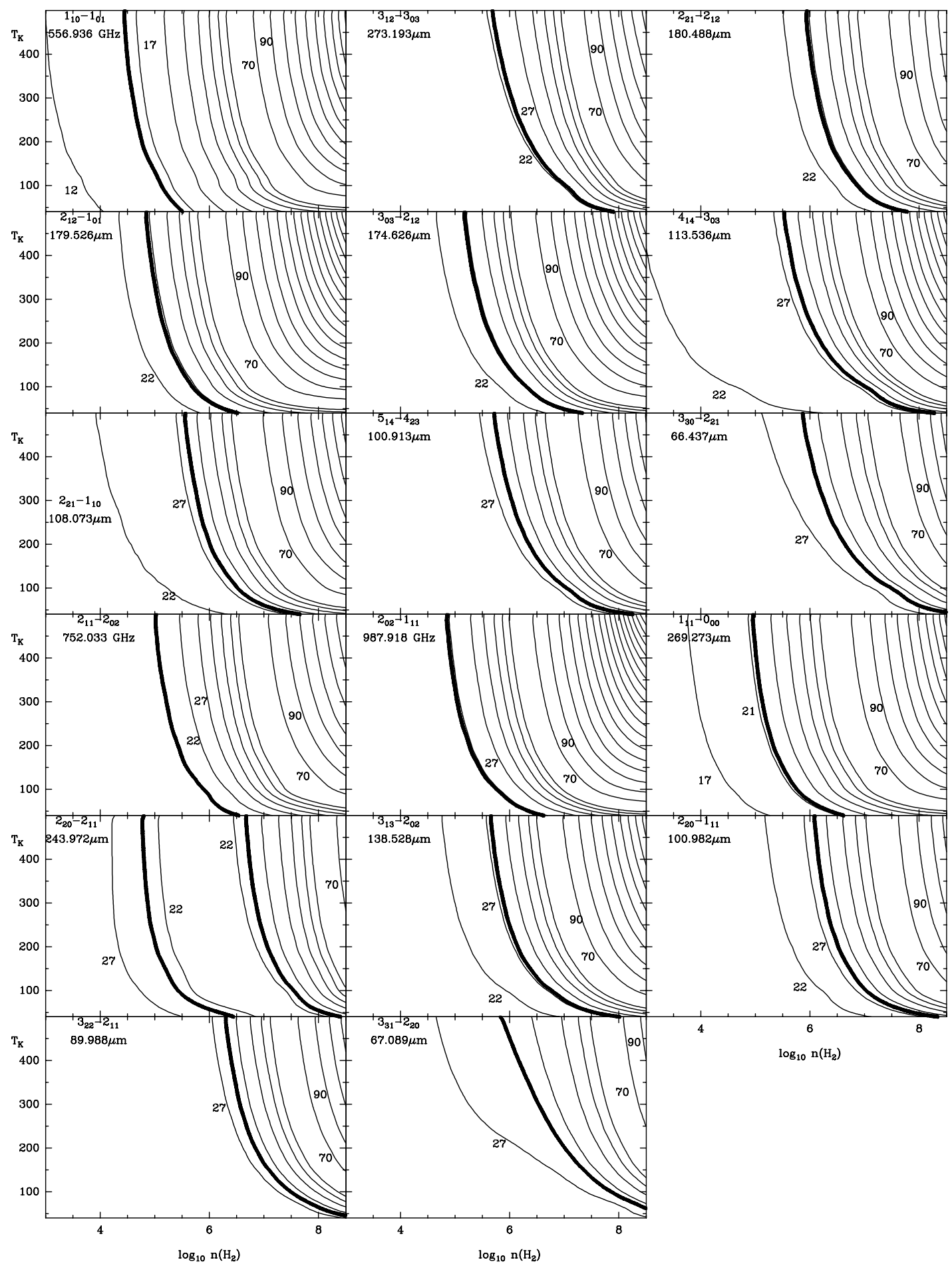

FIG. 6.-LVG excitation models of different kinetic temperature and density for several water vapor rotational lines. The column density of ortho- $\mathrm{H}_{2} \mathrm{O}$ is $1.8 \times$ $10^{16} \mathrm{~cm}^{-2}$ and that of para- $\mathrm{H}_{2} \mathrm{O}$ is $0.6 \times 10^{16} \mathrm{~cm}^{-2}$. In each panel, the $\mathrm{H}_{2} \mathrm{O}$ transition and wavelength/frequency is labeled. The thick contour corresponds to the equivalent temperature of the continuum source $\left(T_{c}\right)$. Hence, to the left of this contour, lines will be in absorption $\left(T_{\mathrm{ex}}<T_{c}\right)$.

$1_{10}-1_{01}$ line observed in the $180 \mathrm{pc}$ "molecular ring" around the GC between $v_{\text {LSR }} \sim+80$ and $120 \mathrm{~km} \mathrm{~s}^{-1}$ (Neufeld et al. 2003). For the density conditions derived for the Sgr B2 velocity range and for the line -of-sight clouds, the $557 \mathrm{GHz}$ line is correctly predicted in absorption. A similar behavior is expected for the para- $\mathrm{H}_{2} \mathrm{O} 1_{11}-1_{00}$ line at $\sim 269.3 \mu \mathrm{m}(\sim 1113 \mathrm{GHz})$ that will be observed by future heterodyne instruments such as HIFI/Herschel.

Among all the moderate excitation para- $\mathrm{H}_{2} \mathrm{O}$ lines $\left(E_{l}<450 \mathrm{~K}\right)$, only the $3_{13}-2_{20}$ appears in the millimeter domain. Contrary to other $\mathrm{H}_{2} \mathrm{O}$ maser lines accessible from ground-based telescopes, relatively low $T_{k}$ and density are required for the $3_{13}-2_{20}$ line inversion. These conditions allowed the first detection of extended water emission in Orion (Cernicharo et al. 1994), and a strong dependence of the emission with $T_{k}$ was revealed. Hence, the 183.31 GHz line could be an excellent tracer of the warm gas in molecular clouds.

We have used the same LVG model to analyze the $183.31 \mathrm{GHz}$ line inversion mechanism that produces extended emission in 


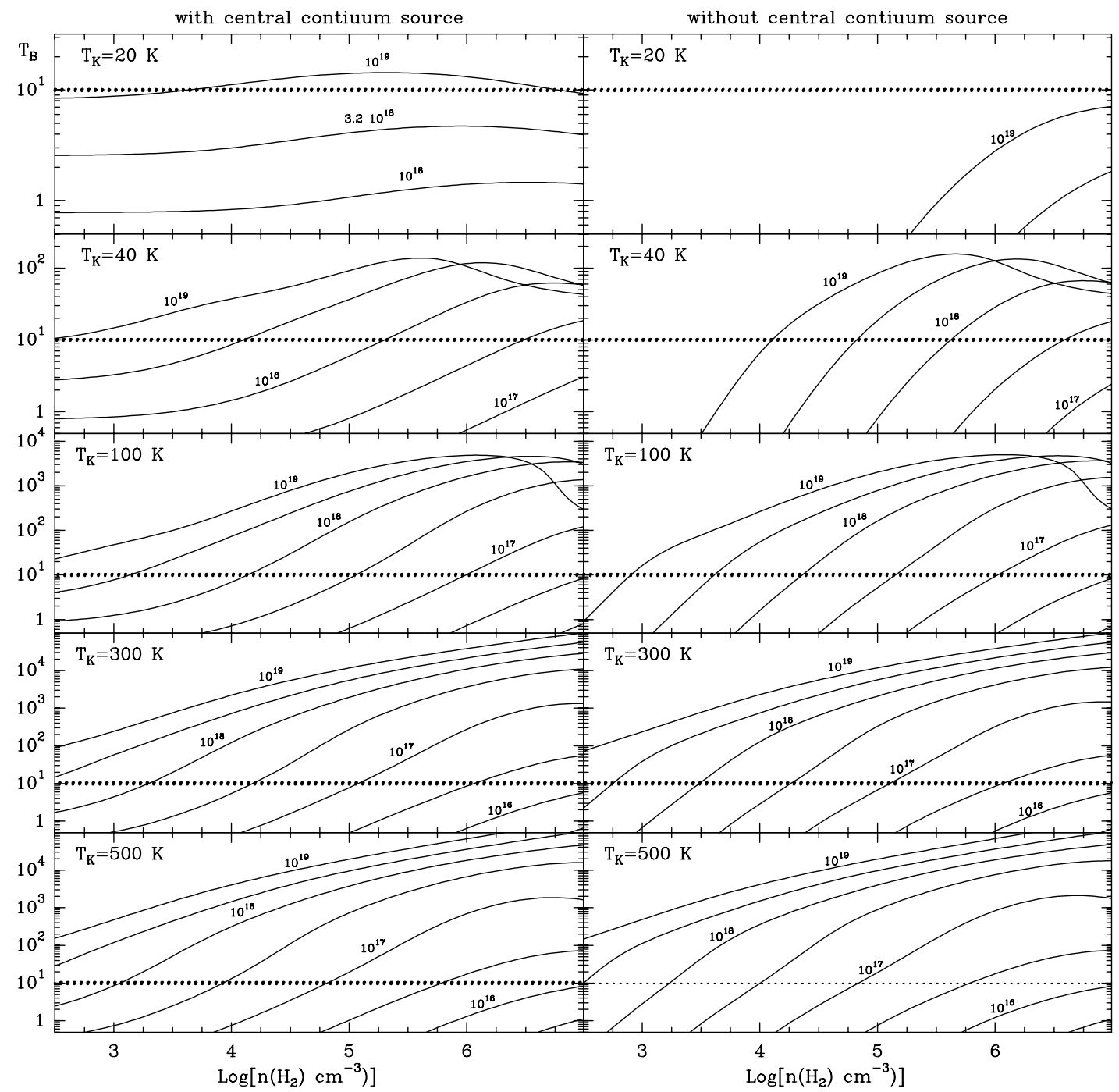

FIG. 7.-LVG excitation models for the $3_{13}-2_{20}$ maser transition of para- $\mathrm{H}_{2}^{16} \mathrm{O}$ at $183.31 \mathrm{GHz}$. Models with and without a central continuum source (described as a graybody with an opacity of 2.5 at $80 \mu \mathrm{m}$, with a dust opacity law given by $\tau_{\lambda}=\tau_{80}[80 / \lambda(\mu \mathrm{m})]$, and a dust temperature of $30 \mathrm{~K}$ ) have been considered. For low temperature and low densities, infrared pumping seems to be efficient to increase the emerging flux of the $183.3 \mathrm{GHz}$ line. In addition, the maser amplifies the continuum. Hence, the resulting flux depends on the assumed opacity at $80 \mu \mathrm{m}$ and on the opacity law. For higher kinetic temperatures and densities collisional pumping of the maser dominates and the difference between the two cases, with and without central continuum source, is less important. For a given kinetic temperature $(20-500 \mathrm{~K})$, the expected $T_{B}$ as a function of the $\mathrm{H}_{2}$ density is shown in each panel for different column densities of para- $\mathrm{H}_{2}^{16} \mathrm{O}$. The dashed line indicates the observed brightness temperature in the extended emission. Toward the main condensations, brightness temperatures of $\sim 100 \mathrm{~K}$ are reached. [See the electronic edition of the Journal for a color version of this figure.]

Sgr B2 (Fig. 2). Figure 7 shows different excitation models for the $3_{13}-2_{20}$ transition. Since a fraction of the $183.31 \mathrm{GHz}$ line emission may arise from the warm envelope in front of the far-IR continuum source, models with and without a central continuum source (the same described at the beginning of the section) have been considered. For a given $T_{k}$ (from 20 to $500 \mathrm{~K}$ ), each panel shows computations of different para- $\mathrm{H}_{2} \mathrm{O}$ column densities as a function of $T_{B}$ (in $\mathrm{K}$ ) and $\mathrm{H}_{2}$ volume density. For low temperatures $\left(T_{k}<40 \mathrm{~K}\right)$ and moderate densities $\left(<10^{5} \mathrm{~cm}^{-3}\right)$, the line will be observable for large $N\left(\mathrm{p}-\mathrm{H}_{2} \mathrm{O}\right)$ values only if a continuum source is present. Due to the minor role played by collisions in the pumping of the rotational levels at these temperatures, the line intensity is almost independent of the density. Nevertheless, as the temperature increases, collisions start to be significant, and the expected intensity of the $183.31 \mathrm{GHz}$ line becomes less sensitive to the models with or without the continuum source.

The $183.31 \mathrm{GHz}$ emission is produced by the population inversion of the $3_{13}$ and $2_{20}$ levels. This is due to the different rates (far from the thermalization densities) at which both levels can be populated. The $3_{13}$ level is radiatively connected with the $2_{20}$ and $2_{02}$ lower energy levels. The $A_{i j}$ coefficient for the $3_{13}-2_{02}$ at $\sim 138.5 \mu \mathrm{m}$ is $\sim 10^{4}$ times larger than the $A_{i j}$ of the $3_{13}-2_{20}$ transition. The $\sim 138.5 \mu \mathrm{m}$ line is seen in strong absorption in the ISO LWS spectra (see Fig. 1). The $2_{20}$ level is radiatively connected with the $2_{11}$ and $1_{11}$ lower energy levels. The $2_{20}-1_{11}$ at $\sim 100.9 \mu$ m produces the strongest absorption of para- $\mathrm{H}_{2} \mathrm{O}$ in the far-IR (see Fig. 1). On the other hand, the $3_{13}$ level is radiatively connected with the $3_{22}, 4_{22}$, and $4_{04}$ higher energy levels, while the $2_{20}$ level is connected with the $3_{31}$ (see the $\sim 67.1 \mu \mathrm{m}$ line in Fig. 1) and with the $3_{13}$ higher energy levels. The different radiative pathways and rates (for $n \ll n_{\text {cr }}$ ) at which the $3_{13}$ and $2_{20}$ levels can be populated produce the inversion. LVG models (see Fig. 7) show that this mechanism can be efficient to produce $183.31 \mathrm{GHz}$ line emission in regions of relatively low density.

The observed extended emission at $183.31 \mathrm{GHz}$ has a brightness temperature of $10 \mathrm{~K}$ on average (see Fig. 2). Assuming that it 


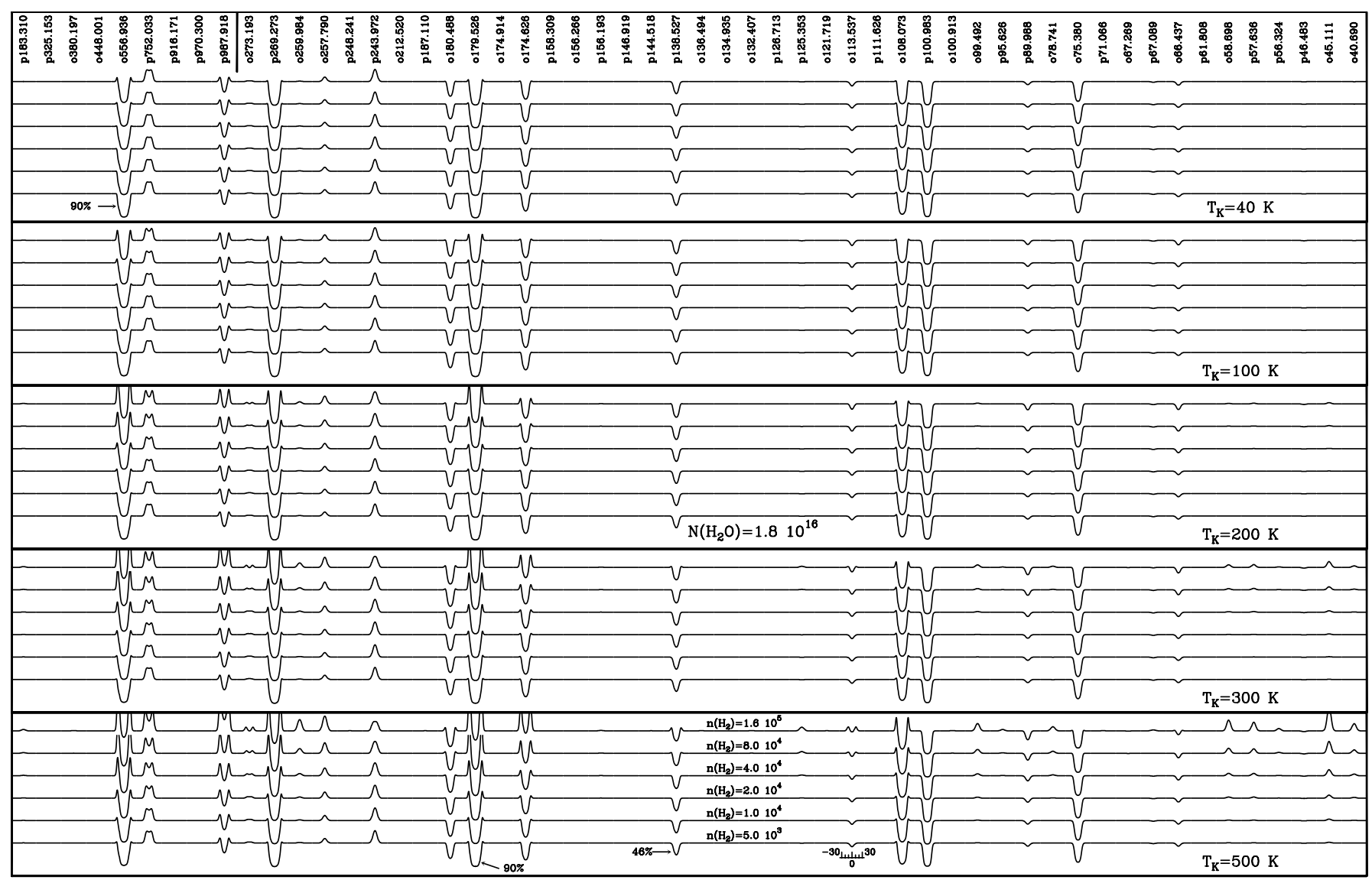

FIG. 8. - Results from selected nonlocal models of $\mathrm{H}_{2} \mathrm{O}$ rotational lines in $\mathrm{Sgr} \mathrm{B} 2(\mathrm{M})$. Results for different kinetic temperatures (from 40 to $500 \mathrm{~K}$ ) and $\mathrm{H}_{2}$ densities (from $5.0 \times 10^{3}$ to $1.6 \times 10^{5} \mathrm{~cm}^{-3}$ ) are shown. Note that for each model, the water abundance changes accordingly with $n\left(\mathrm{H}_{2}\right)$ in order to keep constant the water vapor column density (in this case, $1.8 \times 10^{16} \mathrm{~cm}^{-2}$ ). For the first nine lines, the frequency is given in $\mathrm{GHz}$; for the rest, the wavelength in microns is indicated. The velocity scale is shown at the bottom of the $113.5 \mu \mathrm{m}$ line.

arises from the low-density regions in which the far-IR continuum sources are embedded, with $T_{k}=300-500 \mathrm{~K}$ and $n\left(\mathrm{H}_{2}\right) \sim$ $10^{4} \mathrm{~cm}^{-3}$, we derive a value for $N\left(\mathrm{p}-\mathrm{H}_{2} \mathrm{O}\right)$ of $\gtrsim 5 \times 10^{17} \mathrm{~cm}^{-2}$. This value is higher than the one derived from the far-IR water lines (see $\S 4.2 .2$ ), possibly because the $183.31 \mathrm{GHz}$ line penetrates deeper into this dusty environment. Toward the main condensation, the bulk of the emission seems to arise from the cold $\left(T_{k} \sim 40 \mathrm{~K}\right)$ and dense gas. Under these conditions, a brightness temperature of the line of $\sim 100 \mathrm{~K}$ would translate into a $\mathrm{p}-\mathrm{H}_{2} \mathrm{O}$ column density of $\sim 10^{19} \mathrm{~cm}^{-2}$. However, an important contribution could come from the embedded high temperature and highdensity core condensations where the column density should also be much larger.

\subsubsection{Nonlocal Radiative Transfer Models}

To take into account the radiative coupling between regions with different physical and/or excitation conditions, the radiative transfer has to be treated with nonlocal techniques more sophisticated than the LVG approximation. We have adapted the radiative transfer used in $\S 4.1$ for $\mathrm{CO}$, to the ground vibrational states of ortho- and para- $\mathrm{H}_{2} \mathrm{O}$, respectively. The model includes all the water rotational levels with transitions between $40 \mu \mathrm{m}$ and 183.31 GHz. The level population is computed in statistical equilibrium considering collisional excitation and radiative excitation by line and continuum photons. This is computed consistently assuming that the water molecules and the dust grains are coexistent. The geometry, core + shell dimensions, is the same as that considered in the LVG models. The shell was divided in 41 layers.
The central dust condensation has been modeled with parameters identical to those in $\S 4.2 .1$. We have considered 14 rotational levels $\left(E_{u}<608 \mathrm{~K}\right)$ of orthowater for model with $T_{k}$ below $100 \mathrm{~K}$, and up to 30 rotational levels $\left(E_{\text {up }}<1290 \mathrm{~K}\right)$ for the higher temperature models. We have adopted a turbulence velocity of $8 \mathrm{~km} \mathrm{~s}^{-1}$. The continuum radiation field has been treated as in González-Alfonso \& Cernicharo (1993) by considering a spectral range of $70 \mathrm{~km} \mathrm{~s}^{-1}$ centered on each water transition. Collisional rates have been taken from Green et al. (1993). Obviously, all the water transitions in the continuum core are thermalized to the dust temperature due to the large opacity in the far-IR. The computed line profiles are a result of convolving the brightness temperature with the angular resolution of the LWS detectors $\left(\sim 80^{\prime \prime}\right)$. The resulting spectral resolution of the synthetic water lines is $1 \mathrm{~km} \mathrm{~s}^{-1}$, as no convolution with the spectral resolution has been performed. To test the sensitivity of the model to the physical parameters, models were computed with $N\left(\mathrm{H}_{2} \mathrm{O}\right)$ and $T_{k}$ of $1.8 \times 10^{16}, 9 \times 10^{16}$, and $4.5 \times 10^{17} \mathrm{~cm}^{-2}$, and $40,100,200,300$, and $500 \mathrm{~K}$, respectively, while densities were increased from $5 \times 10^{3}$ to $6.4 \times 10^{5} \mathrm{~cm}^{-3}$ multiplying by 2 in each step. The different nonlocal radiative transfer models for the first two $\mathrm{H}_{2} \mathrm{O}$ column densities are shown in Figures 8 and 9. Models for other column densities have also been run. However, the observed absorption depth is not reproduced for column densities below $10^{16} \mathrm{~cm}^{-2}$. In the $N\left(\mathrm{H}_{2} \mathrm{O}\right)=1.8 \times 10^{17} \mathrm{~cm}^{-2}$ case too many lines would be in emission, contrary to the observations.

The main problem to interpret the $\mathrm{H}_{2} \mathrm{O}$ absorption toward $\mathrm{Sgr}$ $\mathrm{B} 2$ arises from the large opacities of the far-IR water lines, $\sim 10^{3}$, 


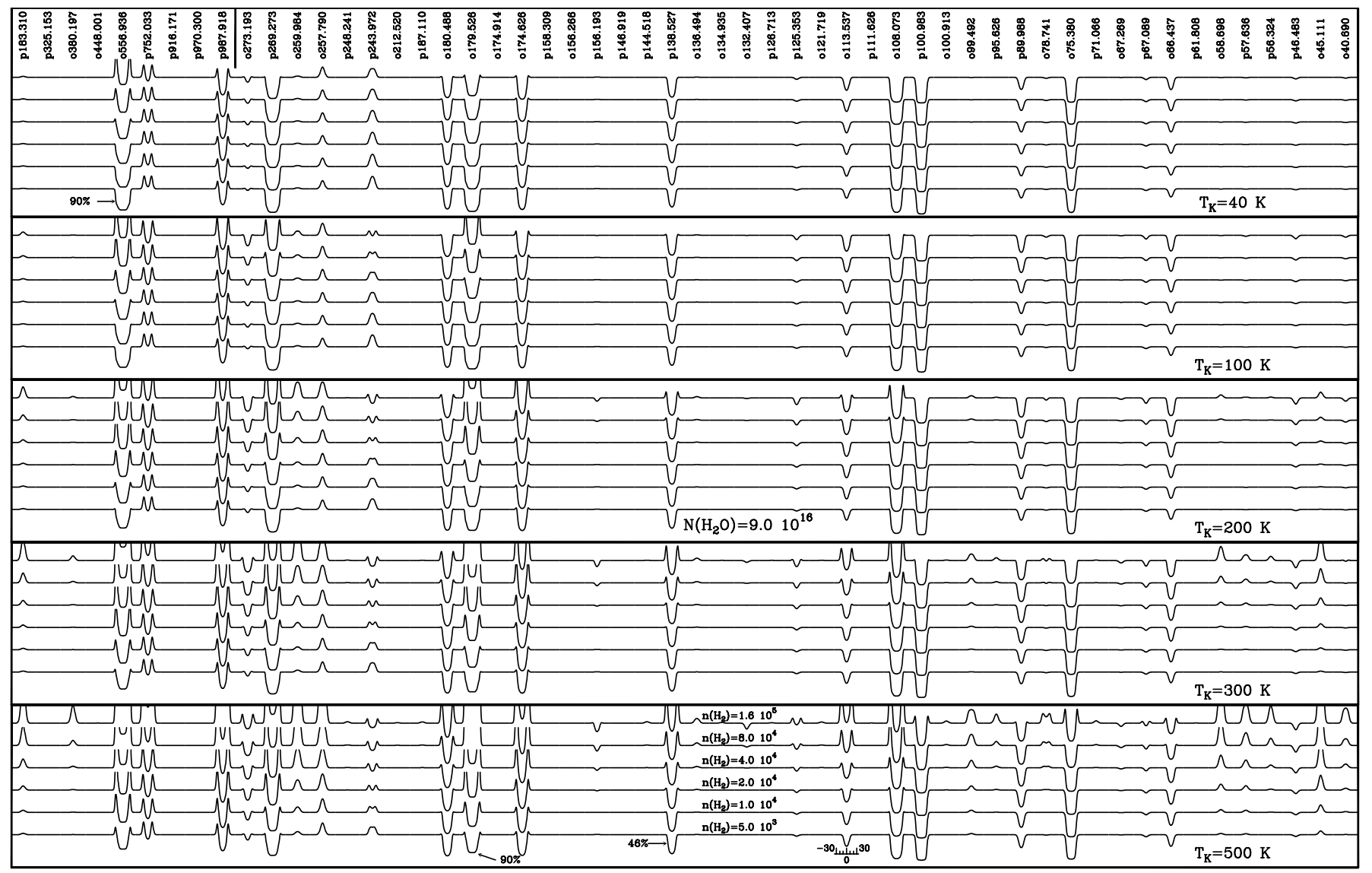

FIG. 9. - Same as in Fig. 8, but for a water vapor column density of $9.0 \times 10^{16} \mathrm{~cm}^{-2}$.

and even $\sim 10^{4}$ for the ortho- $\mathrm{H}_{2} \mathrm{O} 2_{12}-1_{01}$ line at $\sim 179.5 \mu \mathrm{m}$. Under these conditions, many weak water lines have to be detected to constrain the physical conditions and the column density. Therefore, we find that it is difficult to obtain physical parameters from the observation of few far-IR water rotational lines. In addition, as the radiative excitation dominates the population of the far-IR levels, lines with a weak dependence on the dust excitation should be investigated. Taking into account the 14 water lines detected in the far-IR, the nonlocal results imply that models are not very sensitive to the temperature and that the only indication about the column density has to be searched in weak far-IR $\mathrm{H}_{2} \mathrm{O}$ lines or in the $183.31 \mathrm{GHz}$ line. Even so, the far-IR absorption arises in the low-density external layers of gas, while it is very likely that the $183.31 \mathrm{GHz}$ line could have an important contribution from inner and denser regions. Another complication arises from the fact that for models of low $N\left(\mathrm{H}_{2} \mathrm{O}\right)$ values and $T_{k} \lesssim 200 \mathrm{~K}$, it is also difficult to distinguish between different $\mathrm{H}_{2}$ densities (see Fig. 8). Limits to $T_{k}$ and $n\left(\mathrm{H}_{2}\right)$ have to be searched in weak lines below $70 \mu \mathrm{m}$.

The models for high water column densities (Fig. 9) predict absorption lines in the LWS range at $\sim 56.3, \sim 57.6, \sim 58.7$, $\sim 78.7, \sim 99.5, \sim 125.4$, and $\sim 136.5 \mu \mathrm{m}$. At the spectral resolution and sensitivity of the LWS-FP, none of these lines has been detected. This implies that $N\left(\mathrm{H}_{2} \mathrm{O}\right) \leq 4.5 \times 10^{17} \mathrm{~cm}^{-2}$ toward the warm envelope of Sgr B2. The $\sim 136.5 \mu \mathrm{m}$ line (also predicted by the models with large column densities) is contaminated by the absorption produced by the $\mathrm{C}_{3} Q(8)$ rovibrational line, which is also predicted by the models of triatomic carbon (Cernicharo et al. 2000). Some of these lines are predicted (even in emission) by the models with large $N\left(\mathrm{H}_{2} \mathrm{O}\right)$.
Models with $N\left(\mathrm{H}_{2} \mathrm{O}\right)=1.8 \times 10^{16} \mathrm{~cm}^{-2}$ are consistent with ISO detections and upper limits to other ISO lines. Only the $\sim 67.1 \mu \mathrm{m}$ line is weaker than the observations. Therefore, models shown in Figure 8 give a lower limit to the water vapor column density in the outer and warm (300-500 K) envelope. Taking into account the difficulties implied by the $\mathrm{H}_{2} \mathrm{O}$ modeling in the far-IR, we found that $(9 \pm 3) \times 10^{16} \mathrm{~cm}^{-2}$ is the best $\mathrm{H}_{2} \mathrm{O}$ column density to fit the ISO observations (see Fig. 9).

The CO analysis $(\S 5)$, the studies in the far-IR of OH (Goicoechea $\&$ Cernicharo 2002) and the ammonia lines (Ceccarelli et al. 2002), and our CO data, also support that the water absorption lines arise in the warm and low-density $\left(\lesssim 10^{4} \mathrm{~cm}^{-3}\right)$ layer in front of Sgr B2(M). The $\mathrm{H}_{2} \mathrm{O}$ column density derived from ISO observations is below the lower limit of $\left.\mathrm{N}_{(\mathrm{H}} \mathrm{O}\right)$ estimated for the $183.31 \mathrm{GHz}$ line for this component (see $\S 4.2 .1$ ), as it is likely that an important fraction of the $183.31 \mathrm{GHz}$ emission arises from the inner and denser regions closer to Sgr B2 main cores or even from them, as pointed out above. This component, with a mean $\mathrm{H}_{2}$ density of $>10^{5}-10^{6} \mathrm{~cm}^{-3}$, has been traced by the $\mathrm{NH}_{3}$ nonmetastable emission lines, $T_{k} \simeq 100 \mathrm{~K}$ (Hüttemeister et al. 1993), and by the emission of $\mathrm{HC}_{3} \mathrm{~N}$ rotational lines, $T_{k} \simeq 20-40 \mathrm{~K}$ ( Lis $\&$ Goldsmith 1991). The determination of the temperature from millimeter emission lines is also complicated in these regions completely obscured to ISO observations. Therefore, the observed differences in the $3_{13}-2_{02}$ line intensity and shape can be a combination of $N\left(\mathrm{H}_{2} \mathrm{O}\right)$ and/or $T_{k}$ variations across the region.

The results presented in this section have been compared to those obtained from another radiative transfer based on a different approach (Asensio Ramos \& Trujillo Bueno 2003, 2006). This code is a generalization to spherical geometry of the very 
fast iterative Multilevel Gauss-Seidel (MUGA) and Multilevel Successive Overrelaxation (MUSOR) methods developed by Trujillo Bueno \& Fabiani Bendicho (1995) for the case of a twolevel atom and generalized by Fabiani Bendicho et al. 1997) to multilevel atoms in Cartesian geometries. The code also allows the application of the standard Multilevel Accelerated $\Lambda$-iteration (MALI; Olson et al. 1986). The angular information for the calculation of the mean intensity is obtained by solving the radiative transfer equation along its characteristic curves (straight trajectories with constant impact parameter) with the aid of the short-characteristics formal solver with parabolic precision (Kunasz \& Auer 1988). The statistical equilibrium equations are linearized with the aid of the preconditioning scheme developed by Rybicki \& Hummer $(1991,1992)$ with the introduction of an approximate $\Lambda$ operator that can be efficiently obtained in the framework of the short-characteristics technique. The convergence rate of the MUGA and MUSOR schemes is equivalent to that obtained with the introduction of a nonlinear $\Lambda$ operator, with the advantage of not being necessary neither to calculate nor to invert such a nonlinear operator. Interestingly, the time per iteration is similar to that obtained for the standard $\Lambda$-iteration or the MALI method. The computing time for the MUGA scheme is reduced by a factor of 4 with respect to MALI, while the MUSOR scheme leads to an order of magnitude of improvement in the total computing time with respect to MALI.

The calculations have been performed with the same geometry, the same molecular data (collisional and radiative transitions), and the same physical conditions of the previously described nonlocal code. The emerging line profiles from both codes are very similar, with differences below $2 \%-3 \%$. Both codes predict lines in absorption/emission for the same physical conditions, with identical spectral shapes in the cases where reemission is found in the "line wings." This test of consistency allows us to be very confident in the results presented in Figures 8 and 9 , since they were obtained with numerical methods based on completely different approaches.

\section{THE WARM ENVELOPE: SHOCKS, PDRS, OR XDRS?}

The $\mathrm{H}_{2} \mathrm{O}(\mathrm{OH})$ column densities derived from ISO observations in the warm envelope are within an order of magnitude of the shock model predictions for the same low-density region (Flower et al. 1995). Nevertheless, $C$ shocks have been invoked to explain the heating of large amounts of warm gas in the GC and also in the warm envelope (Wilson et al. 1982; Martín-Pintado et al. 1997). In addition, the shock models of Flower et al. (1995) correctly reproduce the observed column densities of $\mathrm{N}$-bearing species such as $\mathrm{NH}_{2}$ and $\mathrm{NH}_{3}$ that could ultimately be related to the dust grain chemistry (G04).

A qualitative explanation came from the observation of large $\mathrm{OH}$ column densities $\left(\mathrm{H}_{2} \mathrm{O} / \mathrm{OH} \sim 2-4\right)$ in the warm envelope. According to Goicoechea \& Cernicharo (2002), if a far-UV radiation field illuminates the outer regions of the cloud, water molecules could be photodissociated, producing an enhancement of the $\mathrm{OH}$ abundance expected only from its formation through $\mathrm{H}_{3} \mathrm{O}^{+}$dissociative recombination. The presence of such an extended far-UV radiation field with $G_{0} \sim 10^{3}-10^{4}$ is inferred from the [O III], [N III], [N II], [C II], and [O I] extended line emission (G04). Therefore, an important contribution from $\mathrm{H}_{2} \mathrm{O}$ photodissociation may explain the observed $\mathrm{H}_{2} \mathrm{O} / \mathrm{OH}$ abundance ratio in the external layers of the envelope.

To investigate in more detail the O-chemistry in the envelope, we have run several photochemistry calculations that include depth-dependent photodissociation, $\mathrm{H}_{3} \mathrm{O}^{+}$dissociative recombination, and neutral-neutral reactions. We have used the latest

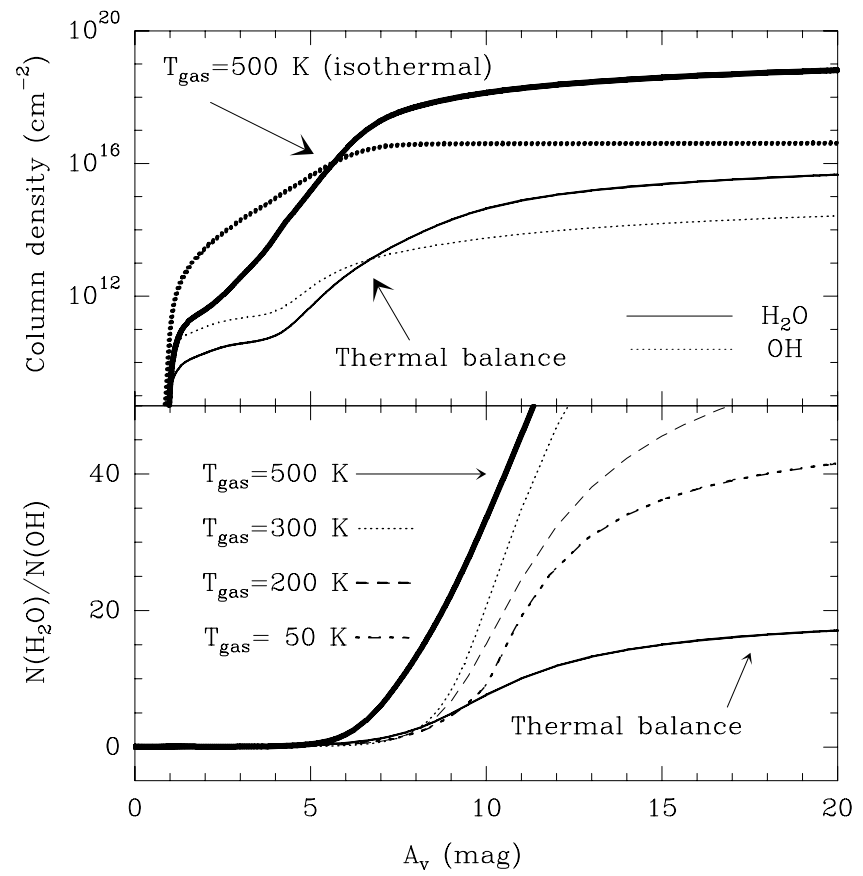

FIG. 10.- Selected PDR models in which $G_{0}=5 \times 10^{3}$ and $n_{\mathrm{H}}=5 \times 10^{3} \mathrm{~cm}^{-3}$. Top: $\mathrm{H}_{2} \mathrm{O}$ and $\mathrm{OH}$ column densities as a function of the visual extinction for an isothermal model with $T_{\text {gas }}=500 \mathrm{~K}$ and for a model in which the full thermal balance is solved at each depth. Bottom: $\mathrm{H}_{2} \mathrm{O} / \mathrm{OH}$ column density ratio as a function of the visual extinction for different isothermal models with $T_{\text {gas }}=500$, 300,200 , and $50 \mathrm{~K}$ and for full thermal balance. [See the electronic edition of the Journal for a color version of this figure.]

version of the public and available PDR model $^{6}$ of Le Bourlot et al. (1993). The model does not include oxygen grain surface chemistry, but it is consistent with the low densities and high temperatures found in the Sgr B2 envelope. Following G04), we assume a $G_{0}=5 \times 10^{3}$ radiation field and a $n_{\mathrm{H}}=n(\mathrm{H})+$ $2 n\left(\mathrm{H}_{2}\right)=5 \times 10^{3} \mathrm{~cm}^{-3}$ density. Assuming that the outer gas layers of the Sgr B2 envelope are directly illuminated by such a radiation field, the model solves the UV transfer and the chemistry up to $A_{V}=20 \mathrm{mag}$. As a reference model we solve the thermal balance explicitly for an initial gas temperature of $500 \mathrm{~K}$. The resulting $\mathrm{H}_{2} \mathrm{O}$ and $\mathrm{OH}$ column densities and the $\mathrm{H}_{2} \mathrm{O} / \mathrm{OH}$ ratio are shown in Figure 10 as a function of the visual extinction through the cloud. This pure PDR can only maintain the temperature above $\sim 100 \mathrm{~K}$ in the first 2 mag of the cloud, where the UV radiation efficiently photodissociates $\mathrm{H}_{2} \mathrm{O}$ to form $\mathrm{OH}$. This results in a low $\mathrm{H}_{2} \mathrm{O} / \mathrm{OH}$ ratio. Inside the cloud, the $\mathrm{H}_{2} \mathrm{O}$ and $\mathrm{OH}$ production is rapidly dominated by $\mathrm{H}_{3} \mathrm{O}^{+}$dissociative recombination, and the $\mathrm{H}_{2} \mathrm{O} / \mathrm{OH}$ ratio tends to a constant value that basically depends on the assumed branching ratio $f_{\mathrm{H}_{2} \mathrm{O}}$ (we have taken $f_{\mathrm{H}_{2} \mathrm{O}}=0.25$ ).

At least two different scenarios can produce large column densities of gas at high $T_{k}$. The first one is the presence of several clumpy PDRs within ISO's beam. In this scenario, far-IR $\mathrm{H}_{2} \mathrm{O}$ and $\mathrm{OH}$ observations will only trace the PDR-clump surfaces. The second one is the presence of shocks. It is very likely that widespread low-velocity shocks can locally heat the gas within the envelope and preserve a fraction of the molecular gas with temperatures up to $\sim 500 \mathrm{~K}$ (Flower et al. 1995), but the same is possible in the clumpy PDR scenario. With these temperatures neutral-neutral reactions play an important role in the chemistry. To simulate this situation, we have run several isothermal models

\footnotetext{
${ }^{6}$ See http://aristote.obspm.fr/MIS.
} 
with temperatures ranging from 50 to $500 \mathrm{~K}$. Some selected results are also shown in Figure 10. From these models it is clear that both $N\left(\mathrm{H}_{2} \mathrm{O}\right)$ and $N(\mathrm{OH})$ are clearly enhanced by neutral-neutral reactions (Fig. 10, top). If the temperature is high, these models reproduce the observational values much better. Hence, a significant fraction of warm gas seems to be needed to reproduce the large $\mathrm{H}_{2} \mathrm{O}$ and $\mathrm{OH}$ column densities observed toward Sgr B2. This conclusion agrees with the large temperatures derived from $\mathrm{OH}$ observations in the far-IR (Goicoechea \& Cernicharo 2002).

The gas temperature determines the contribution of neutralneutral reactions (1) and (2). As the temperature of the gas increases, reaction (1) contributes to a enhancement of the $\mathrm{H}_{2} \mathrm{O} / \mathrm{OH}$ ratio (Fig. 10, bottom). Note that a moderate increase of the radiation field (to $G_{0}=10^{4}$ ) shifts the $\mathrm{H}_{2} \mathrm{O} / \mathrm{OH}$ curve to larger $A_{V}$ inside the cloud until photodissociation becomes less important. In the shielded regions of the cloud, the predicted column densities are similar to those with $G_{0}=5 \times 10^{3}$.

The $\mathrm{H}_{2}$ column density (or $A_{V}$ ) responsible for the warm gas observed from far-IR absorption lines (the location of the species in optical depth) has been traditionally difficult to establish. A comparison between the observational $\mathrm{H}_{2} \mathrm{O} / \mathrm{OH}$ ratio and photochemistry models shows that the bulk of the $\mathrm{H}_{2} \mathrm{O} / \mathrm{OH}$ absorption must arise from the surface of Sgr B2 (a maximum $A_{V}$ of $\sim 5-10 \mathrm{mag}$ ), in agreement with the large opacities derived from the radiative transfer models. Even assuming a homogeneous surface cloud, most of the water vapor will arise from $A_{V}<10 \mathrm{mag}$ if $T_{k} \sim 500 \mathrm{~K}$, while $\gtrsim 50 \%$ of water can be at $A_{V}>10$ mag if $T_{k} \sim 300 \mathrm{~K}$. Therefore, an accurate description of the thermal structure of the cloud (with far-IR diagnostics tracing the same gas) will be needed to establish more detailed conclusions. Still, in an inhomogeneous medium, several PDR-like clump surfaces locally heated to $T_{k} \sim 300-500 \mathrm{~K}$ by low-velocity shocks could be entirely responsible for the far-IR $\mathrm{H}_{2} \mathrm{O} / \mathrm{OH}$ absorption.

Taking into account the uncertainties implied in the determination of column densities in the envelope, we take $\chi\left(\mathrm{H}_{2} \mathrm{O}\right) \simeq$ $(1-2) \times 10^{-5}$ as a lower limit. There is a determination of HD column density toward Sgr B2 of $\sim 10^{18} \mathrm{~cm}^{-2}$, which translates into $N\left(\mathrm{H}_{2}\right) \sim 10^{24} \mathrm{~cm}^{-2}$ (Polehampton et al. 2002). However, taking into account the huge absorption by dust at $112 \mu \mathrm{m}$ $(\tau \sim 4)$, it is unlikely that in this HD column density the cold gas, where high column densities are expected, is accounted for. We derive (see Goicoechea \& Cernicharo 2002) that the $\mathrm{H}_{2}$ column density in the warm gas is $\sim 10^{22} \mathrm{~cm}^{-2}$. Moreover, the $183.31 \mathrm{GHz}$ observations imply that the water abundance is at least an order of magnitude lower in the core regions.

However, we underline the importance of further detection of weak $\mathrm{H}_{2} \mathrm{O}$ lines and/or lines with a lesser dependence from the dust emission to refine the models and better constrain the physical parameters of the region (e.g., the temperature) that also determine much of the chemistry. Especially important will be the input of the Herschel/HIFI observations for water lines below $\sim 2 \mathrm{THz}$.

Another difficult problem is to place the origin of the FUV radiation field revealed by the fine-structure line observations in the region (G04). In principle, FUV photons could arise from the massive stars near the Sgr B2(M) core and/or from another stellar population within the envelope itself (not resolved yet by observations). The permeating effect of the radiation field will be determined by the clumpiness and inhomogeneity of the medium surrounding the stars, and by the energy of the stellar photons. In addition, X-rays observations could complement this scenario of widespread low-velocity shocks and UV radiation. Energetic EUV and/or X-ray photons can penetrate deeper in the neutral cloud than FUV photons and thus could also play a role in the chemistry, as they can also induce many photoionization and photodissociation processes. The correlation found in the Sgr B complex between the $6.4 \mathrm{keV} \mathrm{Fe}{ }^{0}$ line and the $\mathrm{SiO}$ emission could also indicate that the X-ray sources also drive the shocks in the region (Martín-Pintado et al. 2000).

Gas temperatures in XDRs can easily reach $T_{k} \sim 300-500 \mathrm{~K}$ because of the more efficient gas heating by X-ray-induced photoelectrons from the gas (and not from dust grains as in PDRs). Under these conditions, neutral-neutral reactions dominate the chemistry, and especially $\mathrm{OH}$ reaches large abundances. However, XDR models predict low $\mathrm{H}_{2} \mathrm{O} / \mathrm{OH}<0.1$ abundance ratios (Maloney et al. 1996), and thus they cannot be the dominant scenario explaining the far-IR water and $\mathrm{OH}$ lines toward Sgr B2 (Goicoechea \& Cernicharo 2002).

$\mathrm{Sgr} \mathrm{B} 2$ shows diffuse emission in the $\mathrm{K} \alpha$ line of $\mathrm{Fe}^{0}$ at $6.4 \mathrm{keV}$ (Murakami et al. 2001). A dozen compact X-ray sources have been detected within Sgr B2 cloud, and they may explain the whole X-ray emission found in the region. Many of the detected $\mathrm{X}$-ray sources are not associated with radio ( $\mathrm{H}$ II regions created by massive stars) nor with IR sources. According to these observations, the intrinsic X-ray luminosity toward Sgr B2(M) is $L_{\mathrm{X}} \lesssim$ $10^{35} \mathrm{ergs} \mathrm{s}^{-1}$, which translates into a X-ray flux incident on molecular gas of $F_{\mathrm{X}} \lesssim 0.1-0.001 \mathrm{ergs} \mathrm{cm}^{-2} \mathrm{~s}^{-1}$ (assuming $\sim 0.1-$ $1 \mathrm{pc}$ from the X-ray source). Hence, the X-ray field within Sgr $\mathrm{B} 2(\mathrm{M})$ is in the low tail, or even weaker, than those studied by Maloney et al. (1996). As noted by G04, the low [O I] $63 \mu \mathrm{m} /[\mathrm{C}$ II $]$ $158 \mu \mathrm{m}$ and ([O I] $63+\left[\mathrm{C}_{\mathrm{II}}\right]$ 158)/FIR intensity ratios observed in the region favor a dominant PDR origin for these lines. Both shock (Draine et al. 1983) and XDR (Maloney et al. 1996) models predict larger intensity ratios.

\section{SUMMARY}

We have carried out far-IR observations of several thermal absorption lines of water vapor toward Sgr B2(M) and have mapped the $183.31 \mathrm{GHz}$ water line around the main dust condensations of the complex. The main conclusions of this work are the following:

1. The detected water absorption lines are very opaque and arise from the warm envelope around Sgr B2(M). The observation of the $\mathrm{CO} J=7-6$ line and the lack of far-IR CO lines at ISO's sensitivities ( $3 \sigma$ limits below $\sim 2 \times 10^{-18} \mathrm{~W} \mathrm{~cm}^{-2}$ ) imply that the density of such a layer is $n\left(\mathrm{H}_{2}\right) \sim 10^{4} \mathrm{~cm}^{-3}$. The para$\mathrm{H}_{2} \mathrm{O} 3_{13}-2_{02}$ line at $183.31 \mathrm{GHz}$ shows $\sim 40^{\prime \prime} \times 40^{\prime \prime}$ extended emission around Sgr B2(M) and $\sim 40^{\prime \prime} \times 20^{\prime \prime}$ around Sgr B2(N). This is the first observation of that line in a GC source and represents further evidence that water vapor is extended in warm molecular clouds.

2. LVG and nonlocal radiative transfer calculations have been carried out to extract the water vapor abundance and to constrain the physical parameters of the absorbing/emitting regions. Because of the radiative excitation by dust photons, the far-IR water lines are not very sensitive to $T_{k}$. Taking into account the analysis of the related species $\mathrm{OH}$ (Goicoechea \& Cernicharo 2002), the water absorption must arise from warm gas at similar temperatures, i.e., from 300 to $500 \mathrm{~K}$. For this warm envelope, we found $N\left(\mathrm{H}_{2} \mathrm{O}\right)=(9 \pm 3) \times 10^{16} \mathrm{~cm}^{-2}$. An important fraction of the 183.31 GHz emission arises from the inner, denser, and colder gas located closer to the main cores. We estimate a water abundance of a few times $10^{-7}$ in the denser regions.

3. Photochemistry models show that a component of warm gas, $\sim 300-500 \mathrm{~K}$, is needed to activate the neutral-neutral reactions and reproduce the large $\mathrm{H}_{2} \mathrm{O}$ and $\mathrm{OH}$ column densities observed in the envelope. We show that $\mathrm{OH}$ and $\mathrm{H}_{2} \mathrm{O}$ far-IR 
observations toward Sgr B2 are surface tracers of the cloud (a maximum $A_{V}$ of 5-10 mag). We found $\chi\left(\mathrm{H}_{2} \mathrm{O}\right) \simeq(1-2) \times 10^{-5}$ in these regions. Although irradiated by FUV, and possibly more energetic photons, affecting the $\mathrm{H}_{2} \mathrm{O} / \mathrm{OH}$ ratio in the outermost layers, a clumpy structure for the PDR is needed. Alternatively, low-velocity shocks could maintain the gas heating through the envelope.

Due to the complexity of Sgr B2 (and also of the GC ISM as a whole), a multiple scenario is needed to explain the modest angular resolution far-IR observations. The input of chemical models, and higher sensitivity and larger spatial resolution
far-IR observations, will lead to a better understanding of the GC environment.

We thank Spanish DGES and PNIE for funding support under grants PANAYA2000-1784, ESP2001-4516, AYA2002-10113-E, ESP2002-01627, AYA2003-02785-E and AYA2004-05792. CSO observations were supported by NSF grant AST-9980846. J. R. G. was also supported by the French Direction de la Recherche in the latest stages of the work. We thank J. Le Bourlot for useful suggestions and for his help with the PDR model.

\section{REFERENCES}

Asensio Ramos, A., \& Trujillo Bueno, J. 2003, in ASP Conf. Ser. 288, Stellar Atmosphere Modeling, ed. I. Hubeny, D. Mihalas, \& K. Werner (San Francisco: ASP), 335

- 2006, in EAS Publ. Ser. 18, Radiative Transfer and Applications to Very Large Telescopes, ed. Ph. Stee. (Les Ulis: EDP Sciences), 25

Bergin, E. A., et al. 2000, ApJ, 539, L129

Ceccarelli, C., et al. 2002, A\&A, 383, 603

Cernicharo, J. 1999, Ap\&SS, 263, 175

Cernicharo, J., Bachiller, R., \& González-Alfonso, E. 1996, A\&A, 305, L5

Cernicharo, J., Goicoechea, J. R., \& Caux, E. 2000, ApJ, 534, L199

Cernicharo, J., González-Alfonso, E., Alcolea, J., Bachiller, R., \& John, D. 1994, ApJ, 432, L59

Cernicharo, J., Pardo, J. R., González-Alfonso, E., Serabyn, E., Phillips, T. G., Benford, D. J., \& Mehringer, D. 1999, ApJ, 520, L131

Cernicharo, J., Thum, C., Hein, H., John, D., Garcia, P., \& Mattioco, F. 1990, A\&A, 231, L15

Cernicharo, J., et al. 1997, A\&A, 323, L25

Cheung, A. C., Rank, D. M., Townes, C. H., Thornton, D. D., \& Welch, W. J. 1969, Nature, 221, 626

Clegg, P. E., et al. 1996, A\&A, 315, L38

Comito, C., Schilke, P., Gerin, M., Phillips, T. G., Zmuidzinas, J., \& Lis, D. C. 2003, A\&A, 402, 635

de Graauw, T., et al. 1996, A\&A, 315, 49

Draine, B. T., Roberge, W. G., \& Dalgarno, A. 1983, A\&A, 264, 485

Fabiani Bendicho, P., Trujillo Bueno, J., \& Auer, L. H. 1997, A\&A, 324, 161

Flower, D. R. 2001, J. Phys. B, 34, 2731

Flower, D. R., Pineau des Forets, G., \& Walmsley, C. M. 1995, A\&A, 294, 815

Goicoechea, J. R., \& Cernicharo, J. 2001, ApJ, 554, L213

. 2002, ApJ, 576, L77

Goicoechea, J. R., Rodríguez-Fernández, N. J., \& Cernicharo, J. 2004, ApJ, 600,214

González-Alfonso, E., \& Cernicharo, J. 1993, A\&A, 279, 506

González-Alfonso, E., Cernicharo, J., Alcolea, J., \& Orlandi, M. A. 1998, A\&A, 334, 1016

Gordon, M. A., Berkermann, U., Mezger, P. G., Zylka, R., Haslam, C. G. T., Kreysa, E., Sievers, A., \& Lemke, R. 1993, A\&A, 280, 208

Green, S., Maluendes, S., \& McLean, A. D. 1993, ApJS, 85, 181

Gry, C., et al. 2003, The ISO Handbook, Volume 3: LWS-The Long Wavelength Spectrometer, ver. 2.1 (ESA SP-1262; Noordwijk: ESA)

Hüttemeister, S., Wilson, T. L., Henkel, C., \& Mauersberger, R. 1993, A\&A, 276,445

Hüttemeister, S., Wilson, T. L., Mauersberger, R., Lemme, C., Dahmen, G., \& Henkel, C. 1995, A\&A, 294, 667

Jacq, T., Walmsley, C. M., Henkel, C., Baudry, A., Mauersberger, R., \& Jewell, P. R. 1990, A\&A, 228, 447

Jensen, M. J., Bilodeau, R. C., Safvan, C. P., Seiersen, K., Andersen, L. H., Pedersen, H. B., \& Heber, O. 2000, ApJ, 543, 764

Kessler, M. F., et al. 1996, A\&A, 315, L27
Kim, S., Martin, C. L., Stark, A. A., \& Lane, A. P. 2002, ApJ, 580, 896

Kooi, J. W., et al. 2000, Int. J. Infrared Millimeter Waves, 21, 1357

Kunasz, P. B., \& Auer, L. H. 1988, J. Quant. Spectrosc. Radiat. Transfer, 39, 67

Langer, W. D., \& Penzias, Arno, A. 1990, ApJ, 357, 477

Le Bourlot, J., Pineau Des Forets, G., Roueff, E., \& Flower, D. R. 1993, A\&A, 267, 233

Lis, D. C., \& Goldsmith, P. F. 1989, ApJ, 337, 704 1990, ApJ, 356, 195 1991, ApJ, 369, 157

Maloney, P. R., Hollenbach, D. J., \& Tielens, A. G. G.M. 1996, ApJ, 446, 561

Martín-Pintado, J., de Vicente, P., \& Fuente, A., \& Planesas, P. 1997, ApJ, 482, L45

Martín-Pintado, J., de Vicente, P., Rodríguez-Fernández, N. J., Fuente, A., \& Planesas, P. 2000, ApJ, 356, L5

Martín-Pintado, J., de Vicente, P., Wilson, T. L., \& Johnston, K. J. 1990, A\&A, 236, 193

Melnick, G. J., et al. 2000, ApJ, 539, L77

Menten, K. M., Melnick, G. J., \& Phillips, T. G. 1990a, ApJ, 350, L41

Menten, K. M., Melnick, G. J., Phillips, T. G., \& Neufeld, D. A. 1990b, ApJ, 363, L27

Murakami, H., Koyama, K., \& Maeda, Y. 2001, ApJ, 558, 687

Neufeld, D. A., Bergin, E. A., Melnick, G. J., \& Goldsmith, P. F. 2003, ApJ, 590,882

Neufeld, D. A., Lepp, S., \& Melnick, G. J. 1995, ApJS, 100, 132

Neufeld, D. A., Zmuidzinas, J., Schilke, P., \& Phillips, T. G. 1997, ApJ, 488, L141

Neufeld, D. A., et al. 2000, ApJ, 539, L111

Nordh, H. L., et al. 2003, A\&A, 402, L21

Olson, G. L., Auer, L. H., \& Buchler, J. R. 1986, J. Quant. Spectrosc. Radiat. Transfer, 35,431

Pardo, J. R., Serabyn, E., \& Wiedner, M. C. 2005, Icarus, 178, 19

Phillips, T. G., van Dishoeck, E. F., \& Keene, J. 1992, ApJ, 399, 533

Polehampton, E. T., Baluteau, J.-P., Ceccarelli, C., Swinyard, B. M., \& Caux, E. 2002, A\&A, 388, L44

Rybicki, G. B., \& Hummer, D. G. 1991, A\&A, 245, 171 1992, A\&A, 262, 209

Swinyard, B. M., et al. 1998, Proc. SPIE, 3354, 888

Trujillo Bueno, J., \& Fabiani Bendicho, P. 1995, ApJ, 455, 646

Waters, J. W., et al. 1980, ApJ, 235, 57

Williams, T. L., Adams, N. G., Babcock, L. M., Herd, C. R., \& Geoghegan, M. 1996, MNRAS, 282, 413

Wilson, T. L., Ruf, K., Walmsley, C. M., Martin, R. N., Batrla, W., \& Pauls, T. A. 1982, A\&A, 115, 185

Zmuidzinas, J., Blake, G. A., Carlstrom, J., Keene, J., Miller, D., Schilke, P., \& Ugras, N. G. 1995, in ASP Conf. Ser. 73, Airborne Astronomy Symposium on the Galactic Ecosystem: From Gas to Stars to Dust, ed. M. R. Haas, J. A. Davidson, \& E. F. Erickson (San Francisco: ASP), 33 